\title{
Die Begründung
}

\section{derAbstammungslehre.}

Von

\section{Gustav Wolff}

Dr. phil. \& med.

Professor der Psychiatrie an der Universität Basel.

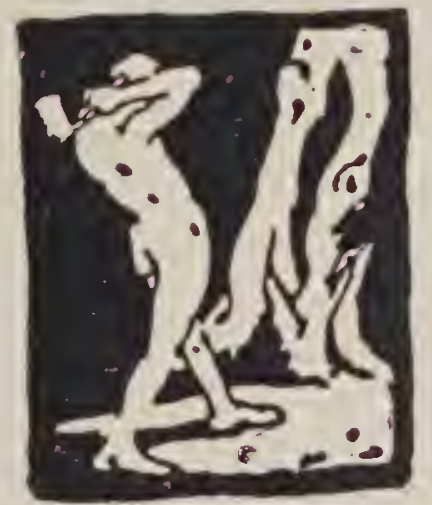

München 1907.

ERNST REINHARDT, Verlagsbuchhandlung,

Jägerstrasse 17. 
Digitized by the Internet Archive in 2020 with funding from University of Toronto 


\section{VORWORT.}

Die Veranlassung zu der nachfolgenden Publikation gab ein Vortrag, den ich am 26. Februar d. J. in der Aula des Basler Museums gehalten habe. Der Vortrag, welcher schon im Aprilheft der „Süddeutschen Monatshefte“ erschienen ist, hat in dem vorliegenden Text nur unwesentliche 'Aenderungen erfahren. Die im Vortrag enthaltene Besprechung des neuerlich wieder in Mode gekommenen Lamarckismus liess es erforderlich erscheinen, auch derjenigen Gestalt eine Besprechung zu widmen, die in der letzten Zeit durch August Pauly dieser Lehre gegeben worden ist. Da diese Besprechung jedoch ihres speziellen Charakters wegen in den auf allgemeine Verständlichkeit berechneten Vortrag sich nicht wohl eingliedern liess, so habe ich dieselbe als zweite Abhandlung dem Vortrag nachfolgen lassen.

Base 1 im April 1907.

\section{Gustav Wolff.}



I.

\section{Hochgeehrte Anwesende!}

Wie kommt dieser Irrenarzt dazu, über die Abstammungslehre $\mathrm{zu}$ sprechen? Mit. solch vernichtendem Argument bin ich schon widerlegt worden, als ich mir erlaubt hatte, Anschauungen über deszendenztheoretische Fragen zu veröffentlichen. Die Wissenschaft huldigt manchmal etwas allzu ergeben dem Prinzip der Arbeitsteilung. Ein Versuch, über den Zaun des Spezialgebietes zu klettern, wird nicht immer mit Wohlwollen vermerkt. Auch die Wissenschaft hat ihre Grenzwächter, dic ihr Amt mit Treue versehen.

Aber hat die Abstammungslehre wirklich so gar keine Beziehungen zur Psychiatrie?

Worin liegt denn das ungeheure Interesse begründet, welches dieser Lehre von allen Seiten entgegengebracht wird? Ist es nur die allgemeine Sehnsucht nach einer Antwort auf die Frage, ob der Bandwurm der Vetter des Regenwurms, ob die Heuschrecke die Tante der Reblaus ist? Das, was die Abstammungslehre zu einem Gegenstand der höchsten Teilnahme aller Gebildeten gemacht hat, sind gewiss nicht die Enthüllungen über Verwandtschaftsverhältnisse von Tieren und Pflanzen, nicht die gelieferten Stammbäume und „Ahnengalerien“, sondern es ist die Antwort, die sie zu geben versucht aut die Frage nach dem Ursprung des Menschen, nach unserer eigenen Herkunft, und zwar nicht nach der Herkunft unserer Nase oder unseres Blinddarms, sondern nach der Herkunft unseres Geistes, unserer Seele, unseres leigentlichen Wesens. Und um diese Lehre, welche die Frage nach der Herkunft des menschlichen Geistes $\mathrm{zu}_{\text {b }}$ beantworten sucht, sollte sich derjenige nicht kümmern dürfen, dessen Arbeitsgebiet die Erkrankungen des menschlichen Geistes bilden?

Die Abstammungslehre nimmt bekanntlich an, dass die Arten der Lebewesen, die in bunter Mannigfaltigkeit unserem 
Blicke sich darbieten, das Produkt eines Entwicklungsvorganges sind, dass die einen Formen aus den andern, im allgemeinen die höheren aus den niederen, durch einen Umwandlungsprozess hervorgegangen sind, und dass vielleicht das ganze Organismenreich auf einen einheitlichen Ursprung zurückzuführen ist. Diese Lehre ist eine durchaus moderne - nicht in dem Sinne, dass sie in der neueren Zeit zum erstenmal ausgesprochen wurde, sondern in dem Sinn, dass sie erst in der Neuzeit zu fast allgemeiner Anerkennung gelangt ist, und dass sie dem modernen Denken ihr Gepräge aufgedrückt hat, dergestalt, dass nicht nur die biologischen Wissenschaften die Abstammungslehre als ihr Fundament betrachten, sondern auch fast alle Disziplinen sich mit ihr auseinanderzusetzen, womöglich auf sie sich zu stützen für notwendig finden.

Was hat frühere Zeiten abgehalten, dem uralten Gedanken der Abstammungslehre ihre Anerkennung zu versagen? Darüber hat sich Kant einmal ausgesprochen. ${ }^{1}$ ) Er weist hin auf „die Uebereinstimmung so vieler Tiergattungen in einem gewissen gemeinsamen Schema, das nicht allein in ihrem Knochenbau, sondern auch in der Anordnung der übrigen Teile zum Grunde zu liegen scheint, wo bewunderungswürdige Einfalt durch Verkürzung e in e $r$ und Verlängerung a nd e re $r$, durch Einwicklung dieser und Auswicklung jener Teile eine so grosse Mannigfaltigkeit von Spezies hat hervorbringen können“, und fügt hinzu: „Diese Analogie der Formen, sofern sie bei aller Verschiedenheit einem gemeinsamen Urbilde gemäss erzeugt zu sein schienen, verstärkt die Vermutung einer wirklichen Verwandtschaft derselben in der Erzeugung von einer gemeinschaftlichen Urmutter, durch die stufenartige Annäherung einer Tiergattung zur andern." Aber Kant sagt weiter: „Eine Hypothese von solcher Art kann man ein gewagtes Abenteuer der Vernunft nennen; und es mögen wenige, selbst von den scharfsinnigsten Naturforschern sein, denen es nicht bisweilen durch den Kopf gegangen wäre. Denn ungereimt ist es eben nicht .... Allein die Erfahrung zeigt davon kein Beispiel.“

Der Mangel an Erfahrung über das Entstehen einer Art aus einer anderen ist es also nach Kant gewesen, der die Forscher früherer Zeiten abhielt, der Abstammungslehre eine wisschenschaftliche Begründung zuzuerkennen.

') Kritik der Urteilskraft. 2. Auflage p. 368. 
Wie ist es nun gekommen, dass diese früher abgelehnte Theorie siebzig Jahre nachdem Kant das Mitgeteilte geschrieben, sozusagen über Nacht alle Geister erobert hat? Sie wissen, dass dies Darwins Werk gewesen ist. Ihm ist es gelungen, durch die von ihm erdachte Selektionstheorie die wissenschaftliche WeIt für die Abstammungslehre zu gewinnen, ihr so festen Boden zu sichern, dass sie stehen blieb, selbst als ihre Stütze wieder fiel. Denn auch heute, wo die Wissenschaft im Begriffe steht, Darwins Schöpfung, die Zuchtwahllehre, wieder aufzugeben, wird es als das bleibende Verdienst Darwins bezeichnet, der Abstammungslehre durch seine, wenn auch falsche Lehre zur allgemeinen Anerkennung verholfen, die Abstammungslehre also bei der Masse durchgebracht zu haben.

Sie kennen alle den Gedanken oder besser die Gedankenlosigkeit der Selektionstheorie, welche das Entstehen mit dem Erhaltenbleiben verwechselt, welche uns zeigt, dass eine schlechtfliegende Taube leichter vom Habicht gefressen wird, als eine gutfliegende, und welche der Welt suggeriert hat, mit der glücklichen Aufdeckung dieser Tatsache sei die Entstehung schnellfliegender Tauben erklärt. Diese Begründung der Abstammungslehre hat zwar in unserem ,empirischen Zeitalter" die Welt beherrscht, sie hat aber gewiss nicht die von Kant empfundene Lücke ausgefüllt. Sie war keine empirische Begründung, sie konnte nicht an der Hand der Erfahrung zeigen, wie Arten in andere sich umwandeln. Wohl war sie ausgegangen von einer Erfahrungstatsache, den Ergebnissen der künstlichen Züchtung von Pflanzen und Tieren. Aber aus diesen Tatsachen konnte sie ja niemals eine empirische Begründung der Entwicklungslehre ableiten. Denn die Tatsachen der künstlichen Züchtung waren viel eher als empirischer Beweis für die Beständigkeit der Arten zu deuten, weil durch sie nur innerhalb ganz bestimmter und nicht überschreitbarer Grenzen eine Formveränderung hervorgerufen werden kann. Gerade die Tatsache, dass wir in manchen Fällen durch künstliche Zuchtwahl verhältnismässig beträchtliche Veränderungen schon innerhalb weniger Generationen erzeugen können, ist eine empirische Grundlage nicht $\mathrm{f} \ddot{\mathrm{u}} \mathrm{r}$ sondern $\mathrm{g}$ e g e n die Darwinsche Lehre von der natürlichen Zuchtwahl; denn die jedesmal sich offenbarende Begrenztheit der Wirkungssphäre der künstlichen Zuchtwahl spricht gegen eine der ersten Voraus- 
setzungen der Selektionstheorie, welche ja eine Plastizität des lebenden Materials fordern muss, die in unbeschränkter Weise nach àllen nur möglichen Richtungen eine stetige und unbegrenzte Veränderung der Organismen durch Selektionsprozesse ermöglicht. Gerade weil die künstliche Zuchtwahl so viel leistet, beweist sie so wenig. Es wäre für die taktische Position der Selektionstheorie viel günstiger, wenn die künstliche Zuchtwahl keine Erfolge aufzuweisen hätte, d. h. wenn die Verfechter auch hier von ihrer einzigen strategischen Methode, der Verschanzung hinter die völlige Unkontrollierbarkeit ihrer Voraussetzungen, Gebrauch machen könnten. Keinesfalls aber konnten die Tatsachen der künstlichen ZuchtwahI dazu dienen, die Lehre von der natürlichen Zuchtwahl empirisch zu begründen, sondern sie konnten nur die Idee der natürlichen Zuchtwahl und deren Erläuterung ermöglichen.

Noch nie war eine Theorie so völlig frei von aller aus Erfahrung geschöpften Begründung. Alle Voraussetzungen der Theorie werden als selbstverständlich vorgetragen und entbehren so sehr einer empirischen Grundlage, dass selbst die erste Voraussetzung der Theorie, das Ueberleben des Passendsten im Kampf ums Dasein, eine Annahme ist, die sich auf keine Beobachtung stützen kann, und gègen deren Berechtigung gerade alles spricht, was die Erfahrung festzustellen vermag. Wohl können wir auf Schritt und Tritt in der Natur Selektionsprozesse beobachten. Wir können überall die längst bekannte Tatsache bestätigt finden, dass von den erzeugten Individuen einer Art nur eine beschränkte Anzahl sich erhalten kann, dass also wirklich fortwährend Selektionsprozesse stattfinden müssen. Aber können wir eine Auslese des Bes s e r e n beobachten?

In einigen Tagen werden die schmelzenden Liebeslieder der Frösche uns ins Freie locken, und ihren mächtigen Willen zum Leben werden wir zum Ausdruck kommen sehen in Gestalt massenhafter schwarzpunktierter Gallertklumpen, welche den Wasserspiegel unserer Tümpel zieren. Jeder dieser schwarzen Punkte will ein Frosch werden, alle können es, alle können es sicher gleich gut. Aber nur eine ganz verschwindende Anzahl, eine kleine Auslese, erreicht diesen Gipfel des Daseins. Der Tümpel trocknet aus, sein Spiegel sinkt, und diejenigen Gallertklumpen, die zufällig an seichteren Stellen schwimmen, geraten aufs 
Trockene. In jedem Frühling können wir eine grosse Menge ausgetrockneter Froschlaichklumpen beobachten. Waren diese Eier minderwertig? Würden weniger gut organisierte Fröschlein aus ihnen hervorgegangen sein, als aus jenen, die ein Zufall ins Nasse gerettet hat? Zu einer solchen Annahme berechtigt uns gewiss keine Erfahrungstatsache.

Und so steht es mit allen Selektionsprozessen, die wir wirklich beobachten können. So weit wir sehen - einige wenige Ausnahmen abgerechnet -, gibt nicht die Zufälligkeit der Ausrüstung, der individuellen Eigenschaften, sondern die Zufälligkeit der Lage, nicht der Vorteil der Organisation, sondern der Situation den Ausschlag, gerade wie bei einem Eisenbahnunglück nicht diejenigen am besten davonkommen, die zufällig die festesten Knochen haben, sondern diejenigen, die zufällig die günstigsten Plätze einnehmen.

Aber dieser schwere Defekt der Darwinschen Theorie, die völlige Unkontrollierbarkeit ihrer Voraussetzungen, er war es nicht, der dem Darwinismus den Hals gebrochen hat. Ueber diese kleine Blösse wäre die Wissenschaft hinweggekommen, ja man hätte gerne aus der Not eine Tugend gemacht, und gerade in diesem Punkte die Stärke der Selektionstheorie erkannt. War doch der Grundsatz: was man nicht kontrollieren kann, das kann man auch nicht widerlegen, von jeher der wissenschaftliche Boden der Zuchtwahlmänner gewesen, die immer ihre Behauptungen am liebsten mit dem begründeten, was man nicht wissen kann. Von diesem Standpunkte aus enthielten ja die meisten Voraussetzungen des Darwinismus eine sieghafte Beweiskraft: sie warer nicht zu widerlegen, weil sie nicht zu prüfen waren.

Was aber geprüft werden konnte, war die Frage, ob die Eigenschaften, welche wir an den Organismen empirisch beobachten, überhaupt entstanden gedacht werden können nach Darwinschen Prinzipien. Die Theorie hatte ja nicht nur zu zeigen, dass Arten auseinander entstehen können, sondern sie hatte auch eine Erklärung zu geben für die ganz bestimmten Eigenschaften oder vielmehr für die ganz bestimmte Eigenschaft, durch welche die Organismen charakterisiert sind. Diese Eigenschaft ist die Zweckmässigkeit, welche sich im Bau und den Verrichtungen der Lebewesen offenbart, und welche das organische Reich in einen für uns bis jetzt unüberbrückbaren Gegensatz stellt zur unbelebten Natur. Diese Zweckmässigkeit der Orga- 
nismen, das grösste Rätsel, welches die Natur uns aufgegeben hat, war von jeher der Lindwurm der Biologie, durch dessen Erlegung ein Ritter nach dem andern die Welt von dem „Alp der Teleologie" zu befreien strebte. Immer wenn es wieder einmal längere Zeit vergeblich versucht worden war, den Lindwurm totzuschlagen, verlegte man sich darauf, ihn totzus a g e n. Man verkündigte, die organische Zweckmässigkeit existiere gar nicht, was auf zwei verschiedene Arten bewiesen werden kann. Entweder man zeigt z. B. einen schief geheilten Knochenbruch und sagt mitleidig lächelnd: „Ach Gott, wie unzweckmässig!" - oder man wird noch gelehrter und sagt: „Die Zweckmässigkeit müssen wir nur infolge unseres eigenartigen Erkenntnisvermögens in die Natur hineinlegen" -, wobei man natürlich vergessen muss, dass unser nämliches $\mathrm{Er}$ kenntnissvermögen die unbelebte Natur anschaut, ohne eine Nötigung zu teleologischer Auffassung zu empfinden.

Als um die Mitte des vorigen Jahrhunderts der Materialismus wieder einmal kräftige Blüten getrieben hatte, da wurde auch das Ableben dieser unangenehmen Zweckmässigkeit feierlich verkündigt. Aber die Auguren hatten doch Mühe, ernst zu bleiben, wenn sie einander ansahen, und man lechzte förmlich nach einer besseren Hinrichtungsmethode. Da trat Darw in auf und zeigte, dass die Zweckmässigkeit nur die notwendige Folge von Ueberproduktion, Variierung, Kampf ums Dasein, Uebrigbleiben des Passenden und Vererbung sei. Das war es, was die Zeit verlangte, und mit stürmischer Begeisterung wurde die neue Lehre aufgenommen, im Siegesrausche um den Erdball getragen, fast ein halbes Jahrhundert als unantastbares Heiligtum gehütet, um dann, nach eingetretener Ernüchterung, in aller Stille begraben zu werden. Allmählich wurde es klar, dass diese Theorie das Zweckmässigkeitsproblem nicht gelöst hatte, schon deshalb nicht, weil sie ja Organismen voraussetzt, also Wesen, welche schon die Eigenschaft der Zweckmässigkeit haben mussten, weil sie also gerade das, was sie erklären will, voraussetzt, und weil es leicht zu beweisen ist, worauf ich im Rahmen meines Vortrages nicht eingehen kann, dass, wenn die Organismen wirklich auf dem Wege einer Entwicklung entstanden sind, die Umwandlung nicht hervorgerufen worden sein kann durch gehäufte zufällige Variierungen, wie Darwin 
annimmt, sondern nur durch bestimmt gerichtete, ein Ziel verfolgende, mit einem Wort $\mathrm{z}$ w e ck mä s s ig e Veränderungen.

Der Darwinismus, welcher die Mission erfüllt hatte, einerseits die Teleologie von der Tagesordnung abzusetzen, andererseits die Entwicklungslehre auf den Schild zu heben, ist so gut wie gefallen, aber seine Werke sind unbesehen stehen geblieben. Man lässt die Selektionstheorie fallen und denkt nicht daran, dass dann auch dasjenige fallen müsste, was durch sie gestützt worden war. Man gibt den Darwinismus auf, nicht aber die Abstammungslehre, die er allein durchgebracht hat. Man sieht den Irrtum ein, lebt aber weiter von șeinen Wirkungen. Denn jedermann weiss, und wer es nicht wissen sollte, der kann es in jedem Lehrbuch der Zoologie, der Botanik, der Paläontologie, der Psychologie und noch in tausend andern Büchern und Broschüren lesen, dass das Hauptverdienst des Darwinismus darin bestand, der vorher von manchen geahnten und geglaubten Abstammungslehre ein wissenschaftliches Fundament gegeben zu haben. Fällt dieses Fundament nun weg, so muss derjenige, der es aus wohlerwogenen Gründen fallen lässt, der unausweichlichen Tatsache ins Auge blicken, dass die Abstammungslehre jetzt ohne dieses wichtigste Fundament dasteht. Und die erste Frage nach der dem Darwinismus gegebenen Absage ist nicht die: welcher Faktor hat nun die Veränderungen der Arten bewirkt? sondern diese: kann die Abstammungslehre auch ohne die Selektionstheorie bestehen? Diese Frag€ wird aber fast nirgends gestellt, ihre Bejahung wird für selbstverständlich gehalten. Das heisst, die Abstammungslehre wird nicht kritisch, sondern dogmatisch festgehalten, und der Darwinismus wird ebenso kritiklos abgelehnt, wie er früher angenommen worden war.

Unter dem Banne des Dogmas stehen noch viele von denen, dije sich äusserlich von demselben befreit haben. Der dogmatische Charakter der Lehre zeigt sich besonders auch darin, dass noch immer mit Vorliebe die Person Darwins in den Vordergrund geschoben und die Angriffe auf seine Lehre als Pietätlosigkeit bezeichnet werden. Und die Mehrzahl derer, welche heute die Zuchtwahllehre verwerfen, erklären dieselbe dennoch für eine der grössten Leistungen und rühmen es als Darwins unvergängliches Verdienst, dass sein ,genialer Irrtum" den Sieg der Abstammungslehre entschieden hat. Wie klein ist der Stand- 
punkt derjenigen, welche sich eines Besitzes freuen können, der nur durch einen Irrtum ihr eigen geworden ist, und die es nach Erkennung dieses Irrtums nicht ihre erste Sorge sein lassen, entweder dieses Gut zurückzuweisen, oder es rechtmässig zu erwerben, um es zu besitzen.

Dass Darwins „Entstehung der Arten“ die Abstammungslehre auch an und für sich, unabhängig von der Selektionstheorie, begründe, ist eine weit verbreitete Meinung, die aber nur von solchen gehegt werden kann, welche die „Entstehung der Arten“ nicht gelesen haben. Dieses Werk sucht nur darzulegen, dass man die Entstehung der Arten durch den Vorgang der Zuchtwahl sich vorstellen könne. Eine Begründung der Abstammungslehre, unabhängig von der Selektionstheorie, wird gar nïcht beabsichtigt. Darwin sucht zwar einige Einwände, die überhaupt gegen die Deszendenztheorie erhoben worden sind, z. B. den auf die Lückenhaftigkeit des paläontologischen Materials gegründeten, zu entkräften, aber eine positive Begründung der Abstammungslehre an und für sich hat er nicht versucht.

Und derselbe Mangel an Kritik offenbart sich in der fortdauernden Ablehnung der Teleologie, die als überwunden gilt, obwohl die einzige scheinbare Widerlegung derselben geifallen ist. Dieser Mangel zeigt sich ferner in der Art und Weise, wie vielfach, nachiem der Darwinismus ins Wanken geraten war, sofort eine ältere, aber längst als ungenügend erkannte Theorie wieder hervorgeholt und für den Darwinismus eingesetzt wurde. Es ist dies die Lehre des französischen Naturforschers Lamarck, der im Beginne des neunzehnten Jahrhunderts eine Begründung der Ábstammungslehre zu geben versucht hatte.

Lamarck war von der jedem einzelnen wohlbekannten Wirkung der Uebung ausgegangen. Wir wissen, dass innerhalb gewisser Grenzen ein Organ, z. B. ein Muskel, durch Gebrauch stärker, durch Nichtgebrauch schwächer wird. Lamarck, welcher voraussetzt, dass die Wirkung der Funktion eines Organes sich auf die Nachkommen vererbt, nimmt nun an, dass der durch viele Generationen bei dem einen Organismus in dieser, bei dem andern in jener Richtung fortgesetzte Gebrauch die Organe in der verschiedensten Weise umgewandelt habe, je nach der Lebensweise, zu welcher die Organismen infolge der veränderten äusseren Bedingungen gezwungen worden waren. 
War ein Tier auf das Laufen angewiesen, so wurden seine Beine immer leistungsfähiger, wie beim Hirsch. Musste sich ein Tier mehr aufs Kriechen verlegen, so wurde die Rumpfmuskulatur immer stärker und die Beine verschwanden nach und nach, wie bei den Schlangen. War es auf dem festen Lande nicht mehr geheuer, so stürzte man sich ins Wasser und schaufeltc mit den Beinen die Fluten Generationen hindurch, so lang€, bis die Beine Flossen waren, wie bei den Walfischen, oder man kletterte auf die Bäume und liess sich mit ausgebreiteten und zappelnden Vorderextremitäten solange herunterfallen, bis nach vielen Generationen die Beine Fallschirme und schliesslich Flügeı ivaren.

Selbst derjenige, der in der glücklichen Lage ist, durch solche Prozesse die Umwandlung der Organismen sich vorstellen zu können, würde diese Theorie vergeblich anzuwenden suchen auf alle diejenigen Organe, deren Funktion lediglich eine passive ist, was schon längst von darwinistischer Seite mit vollem Rechte betont wurde. Unerklärbar blieben also vor allem diejenigen zweckmässigen Einrichtungen, deren Bedeutung nicht darin besteht, den Besitzer zu irgend einer Tätigkeit zu befähigen, sondern die lediglich durch ihr Vorhandensein für den Organismus nützlich sind. Hierher gehören in erster Linie alle Fälle von Mimikry, jener Erscheinung, dass viele Tiere durch Form und Farbe ihre Umgebung nachahmen und dadurch irgend einen Vorteil für ihre Existenz geniessen. Der einfachste Fall von Mimikry ist eine der Umgebung entsprechende Körperfärbung, welche bewirkt, dass ein Tier von seinen Feinden nicht so leicht gesehen wird. Dass es sich hierbei wirklich um zweckmässige Schutzfärbungen handelt, wird besonders klar bei Tieren, deren Farbe sich mit dem Aussehen des Bodens verändert, wie beim Hermelin, das im Sommer braun ist wie die Erde, im Winter weiss wie der Schnee.

Es ist selbstverständlich, dass der Lamarckismus, für solche zweckmässigen Anpassungen keine Erklärung geben kann, denn das Hermelin kann sich niicht geübt haben, weiss oder braun zu sein. Das Weiss- oder Braunsein ist keine Tätigkeit, durch deren Ausübung die Erscheinung graduell verstärkt werden konnte, wie die Muskeln eines Athleten.

Dasselbe gilt von allen denjenigen Einrichtungen, deren Vorhandensein bewirken soll, nicht bloss, dass ein Individuum 
unbemerkt bleibt, sondern, dass es im Gegenteil recht sehr bemerkt wird. Hierher gehören alle Anlockungsmittel. Erwähnen wir aus der Fülle der Beispiele nur das nächstliegende: all die mannigfachen Reize des anderen Geschlechtes, das wir heute zwar nicht mehr das schwache, aber doch, trotz aller Emanzipation, glücklicherweise noch immer das schöne nennen dürfen. Die Schönheit des Weibes hat lediglich durch ihr Vorhandensein dafür zu sorgen, dass die Menschheit nicht ausstirbt. Das Schönsein ist keine Tätigkeit, in der das Weib Generationen hindurch solange sich geübt haben kann, bis es schliesslich so schön war, wie es heute ist. Das Weib kann sich wohl geübt haben in der Kunst, seine Schönheit zur Geltung zu bringen, seine Reize spielen, seinen Zauber wirken zu lassen, aber die Reize selbst'konnten durch diese Tätigkeit keine Steigerung erfahren. Wo zu solcher Steigerung ein Bedürfnis empfunden wurde, war man von jeher auf die Kunsthilfe angewiesen, deren Wirkungen sich aber leider immer noch nicht auf die Nachkommen vererben.

Aber wir wollen uns nicht etwa mit einer Kritik des Lamarckismus aufhalten. Hinweisen wollte ich nur auf die psychologisch interessante Tatsache, wie der Bankerott des Darwinimus die Folge hat, dass mit der grössten Selbstverständlichkeit der vorher für ungenügend erklärte Lamarckismus jetzt hervorgeholt wird. Man macht es offenbar wie jener Mann, der eine Methode erfunden hatte, ohne Kosten immer einen guten Rock auf dem Leibe zu tragen. Er besass zwei Röcke, einer blieb im Schrank, den andern trug er solange, bis er schlechter war als der im Schrank befindliche; dann wurde gewechselt. Auf diese Art trug er immer einen „bessern“ Rock.

Aber wie steht es mit der Abstammungslehre, wenn weder Darwinismus noch Lamarckismus zu ihrer Begründung ausreichen? Ist sie in sich selber fest genug begründet, dass wir ein wissenschaftliches Recht haben, sie anzunehmen, obwohl wir uns keine Vorstellung machen können über die Kräfte, die im Lauf der Geschlechter die Umwandlung der Arten hervorgerufen haben?

Sie sagen mir vielleicht, im Ernste könne doch an der Richtigkeit der Entwicklungslehre niemand zweifeln. Die Ergebnisse der vergleichenden Anatomie der Entwicklungsgeschichte, der Paläontologie usw., seien lauter Tatsachen, die wie aus e in e m 
Munde die Abstammungslehre verkünden. Ja, verehrte Anwesende, so etwas liest man ja sehr häufig, aber auch selten mehr. Wir sind aber berechtigt und verpflichtet, mehr zu verlangen. W a rum sind diese Tatsachen ein Beweis für die Abstammungslehre?

Erinnern wir uns zunächst einmal wieder daran, dass die Tatsachen der vergleichenden Anatomie, der Entwicklungsgeschichte und der Paläontologie schon längst vor Darwins Auftreten den Entwicklungsgedanken nahe gelegt hatten, wie Kants Darlegung beweist, die wir soeben kennen gelernt haben. Dasjenige, was uns diese Disziplinen, also die vergleichende Biologie im weitesten Sinne, lehren, ist im wesentlichen die Tatsache, dass wir die Organismen in mehrere Gruppen ordnen können, deren einzelne Glieder trotz vielfacher Verschiedenheit nach einem gemeinsamen Plane gebaut zu sein scheinen. So fassen wir Fische, Amphibien, Reptilien, Vögel und Säugetiere in die höhere Einteilungsgruppe der "Wirbeltiere" zusammen, weil trotz aller Verschiedenheit eine Aehnlichkeit des Grundplans, nach 'dem alle gebaut zu sein scheinen, nicht zu verkennen ist. Und diese Uebereinstimmung offenbart sich nicht nur im allgemeinen, also etwa darin, dass alle Angehörigen dieser Gruppe (auf Ausnahmen brauchen wir hier nicht einzugehen) eine Wirbelsäule, einen Schädel, vier Gliedmassen besitzen, sondern sie zeigt sich auch im speziellen darin, dass einzelne scheinbar ganz verschiedene Organe, wie die Flügel des Vogels und die Hand des Menschen nach demselben Grundplane gebaut sind.

Wir haben gesehen, dass für die Forscher früherer Zeiten solche Tatsachen zwar genügten, um, wie Kant sagt, den Gedanken der Abstammungslehre sich durch den Kopf gehen zu lassen, nicht aber, um die Lehre als eine wissenschaftlich berechtigte Hypothese oder gar, wie es die heutige Zeit tut, als eine bewiesene Tatsache anzuerkennen. Gerade der Begründer der vergleichenden Anatomie, Cuvier, dem die Tatsachen seiner Wissenschaft gewiss nicht unbekannt gewesen sirı, war ja ein energischer Gegner der Abstammungslehre. Warum beurteilen, wir diese Tatsachen heute so anders? Warum beweisen sie heute dasjenige, woran sie früher höchstens einmal denken liessen? 
Sind die Tatsachen heute zwingender? Die uns bekannten Tatsacher șind allerdings zahlreicher geworden, sie dringen mehr ins einzelne, aber dadurch, dass das Tatsachenmaterial an Extersität gewonnen hat, ist die Intensität seiner Beweiskraft nicht gewachsen. Diese neuen Tatsachen sind es ja auch gar nicht gewesen, deren Beweiskraft die Anerkennung der Abstammungslehre erzwungen hat, sondern erst nachdem die Selektionstheorie unsere Köpfe dazu prädisponiert hatte, erst da wurden jene Tatsachen für zwingend gehalten. Die Kraft, die den Tatsachen an sich inne wohnte, hatte nicht genügt; erst als die ungeheure Suggestionswirkung des Darwinismus hinzukam, da wurden jene Tatsachen mit anderen Augen angesehen. Und unter dieser ungeheuren Suggestionswirkung stehen wir heute noch, stehen auch diejenigen noch, deren Denken die Darwinistisch $€$ Infektion überwunden hat. Die Nachwirkungen auch einer geistigen Epidemie dauern immer länger als die Epidemie selbst.

Wenn wir heute die Tatsachen der vergleichenden Biologie schlechthin für einen Beweis der Abstammungslehre halten, so ist das wirklich nur Nachwirkung der Darwinistischen Suggestion. Diese hat auch in unserer Ueberzeugung die Abstammungslehre durchgebracht, im Lichte der vom Darwinismus her in uns wohnenden Ueberzeugung betrachten wir die Tatsachen, und nicht die Betrachtung der Tatsachen hat uns das Licht dieser Ueberzeugung gebracht.

Suchen wir uns deshalb von aller Suggestionswirkung frei zu machen, und ohne jedes Interesse am Ergebnis die Frage zu prüfen, ob wirklich die Tatsachen der vergleichenden Biologie einen Beweis für die Abstammungslehre bilden.

Weil die Erfahrung uns keinen Aufschluss über die Abstammungslehre gibt, weil die Abstammung einer Art aus der andern nicht direkt beobachtet werden kann, weil wir also von der Richtigkeit der Abstammungslehre nicht durch unmittelbare Anschauung uns überzeugen können, bleibt uns zur Entscheidung der Frage überhaupt nur die Möglichkeit eines Wahrscheinlichkeitsbeweises, genau wie dem Richter da, wo eine vermutete Handlung nicht durch Zeugen beobachtet wurde, nur der Indizienbeweis übrig bleibt. Der Indizienbeweis kann uns nur sagen, dass eine bestimmte Möglichkeit eine mehr oder weniger grosse Wahrscheinlichkeit habe. Aber deswegen kann 
ein Indizienbeweis doch ausserordentlich wertvoll sein, und ebenso wie er unter Umständen den Richter dazu führen darf, eine Verurteilung auszusprechen, so kann der Indizienbeweis den Forscher berechtigen, die Annahme eines vermuteten Vorgangs als eine wissenschaftlich berechtigte anzusehen. Aber wie der Richter, so ist der Forscher verpflichtet, die Kette seines Indizienbeweises mit der allergrössten Genauigkeit zu prüfen.

Der Indizienbeweis beruht im wesentlichen darauf, dass wir eine Reihe zusammenhangsloser Tatsachen durch Annahme eines nicht beobachteten, aber nach unserem Denken und unserer sonstigen Erfahrung möglichen Vorgangs in einen kausalen $\mathrm{Zu}$ sammenhang bringen, also eine Verbindung der beobachteten Tatsachon herstellen, die den sonst durch Erfahrung festgestellten Möglichkeiten entspricht. Es wird z. B. eine Leiche in einer Situation gefunden, aus welcher hervorgeht, dass die betreffende Person von fremder Hand getötet worden ist. Die Zeit des Mordes ist durch gehörte Hilferufe festgestellt worden. Ein des Mordes dringend verdächtiger Mann kommt in Untersuchung, er kann aber nachweisen, dass er eine Viertelstunde nach der Zeit des Mordes an einem Ort sich aufhielt, den er auch durch schnellstes Laufen frühestens in drei Viertelstunden hätte erreichen können. Es ist ferner nachgewiesen, dass keine Gelegenheit vorhanden war, den zweiten Ort per Bahn, zu Wagen oder zu Pferd zu erreichen, es ist überhaupt keine Möglichkeit abzusehen, wie der Verdächtige nach dem andern Ort in so kurzer Zeit hätte gelangen können. Kein Richter würde es auf sich nehmen, auf Grund solcher Indizien einen Menschen zu verurteilen, weil der Indizienbeweis eine klaffende Wunde hat. Es fehlt ihm ein Aufschluss über die Kräfte, die den Verdächtigen von einem Ort nach dem andern gebracht haben können. Der Indizienbeweis verbindet also die beobachteten Tatsachen nicht durch einen angenommenen Vorgang, von dem wir uns wenigstens eine Vorstellung machen können. Ja, wenn im Laufe der Untersuchung bekannt würde, dass am Tage des Mordes in der Nähe der Mordstelle ein Fahrrad abhanden kam, das nach dem Mord in der Nähe des zweiten Ortes gefunden wurde, dann hätte der Indizienbeweis ein anderes Aussehen. Dann wäre der Kausalzusammenhang hergestellt, es wäre wenigstens eine Möglichkeit gezeigt worden, in welcher Weise der ver- 
mutete Vorgang sich abgespielt haben konnte, und erst dadurch würde der Indizienbeweis einen Wert erhalten haben.

Wie steht es nun mit dem Indizienbeweis, den die vergleichende Biologie für die Abstammungslehre führen kann?

Die Indizien für den vermuteten Entwicklungsprozess sind die Uebereinstimmung mehrerer Gruppen von Organismen im Grundplan des Baues. Es fehlt uns aber jede Möglichkeit, uns die Art und Weise, wie verschiedene Arten auseinander stehen können, vorzustellen. Die Annahme der Enitwicklung verbindet die Tatsache jener Uebereinstimmung nicht durch einen präsumierten Vorgang, der unseren Erfahrungstatsachen entspricht, sondern durch einen solchen, über dessen Möglichkeit wir uns auch nicht die leiseste Vorstellung machen können. Diese Tatsache dürfen wir nicht verschleiern, sondern wir müssen unumwunden zugeben, dass in dieser Hinsicht der Indizienbeweis auf recht schwachen Füssen steht.

Wo in aller Welt liegt auch das Zwingende der logischen Ableitung, dass zwei Dinge, die in gewissen Zügen übereinstimmen, von einander abstammen müssen? Man hat schon längst mit Recht darauf hingewiesen, dass ja auch die Mineralien unter einander zu einem System geordnet werden können, bei dem die Angehörigen einzelner Gruppen mehr oder weniger grosse Uebereinstimmungen zeigen, so dass man auch von Verwandtschaft gesprochen hat, natürlich nur im bildlichen Sinne, genau wie es für die Organismen schon zu Linnés Zeiten und früher geschah, ohne dass man dabei an èinen genetischen Zusammenhang dachte.

Stammen vielleicht zwei Häuser, die in ihrer Stilart übereinstimmen, von einander $a b$ ? Höchstens doch nur in der Weise, dass sich aus der Vorstellung des einen Hauses die Vorstellung des andern entwickelt haben kann, eine Art der Entwicklung, welche die heutige Entwicklungslehre gewiss nicht im Auge hat.

Die evidente Beweiskraft, die der vergleichenden Biologie für die Begründung der Abstammungslehre angeblich innewohnt, wird nicht erhöht, durch allerhand Manöver, welche dem grossen Publikum die Sache plausibel machen sollten. Man hat z. B. behauptet, ähnlıch wie unsere heutigen Anschauungen über das Planetensystem ihre glänzendste Bestätigung finden durch Vorhersagungen, die auf Grund unserer allgemeinen Vorstellungen gemacht und dann durch die Erfahrung bestätigt worden sind, 
wie die Entdeckung des Planeten Neptun, so habe man auf der Basis der Entwicklungslehre Dinge vorausgesagt, die dann eine daraufhin gerichtete Untersuchung wirklich gefunden habe. In der Tat hat man schon verschiedene anatomische Verhältnisse vorausgesagt, ich erinnere Sie an Goethe und den berühmten Zwischenkieferknochen. Solche Voraussagen mit nachheriger empirischer Bestätigung kann heutzutage jeder sehr leicht machen. Er braucht nur eine noch nicht in allen anatomischen Einzelheiten durchforschte Tierart vorzunehmen, so kann er auf Grund der systematischen Stellung der betreffenden Art, also auf Grund sonstiger Uebereinstimmungen, genug Dinge voraussagen, die er nachher finden wird. Solche Vorhersagen dürfen aber nicht in Vergleich gestellt werden mit der Vorhersage des Neptun, und für die Entwicklungslehre bringen sie schon deshalb keine Stütze, weil es gar nicht die Entwicklungslehre ist, auf deren Grundlage sie erfolgen. Lediglich die uns empirisch bekannte Uebereinstimmung im anatomischen Grundplan, die wir im allgemeinen bei den Angehörigen eines Typus anzutreffen pflegen, lässt es uns auffallend erscheinen, wenn ein sonst durchgängig angetroffenes Merkmal bei einer Spezies zunächst vermisst wird, und wir können oft mit Erfolg vermuten, dass eine genaue Untersuchung das betreffende Merkmal doch noch finden lässt, wenn auch, wie die Erfahrung uns oft gelehrt hat, nur in embryonalen Spuren. Diese Vorhersagen werden dann aber auf Grund dieser Erfahrungstatsachen gemacht, nicht auf Grund einer etwa auf diesen Tatsachen aufgebauten Theorie. Goethe hatte, als er den Zwischenkieferknochen voraussagte, damals gewiss keinen deszendenztheoretischen Gedanken, selbst wenn er später jemals einen solchen gehegt haben sollte.

Ein ähnliches, auf die urteilslose Masse berechnetes Manöver ist folgendes. Man hat in neuester Zeit die hochinteressante Entdeckung gemacht, dass die Uebereinstimmung einander systematisch nahe stehender Tiere sich in feinen physiologischen Reaktionen zeigt, die mittelst des Blutes solcher Tiere hervorgerufen werden können. Urteilslose Deszendenzfanatiker verkündeten nun mit grossem Geschrei, jetzt sei die Blutsverwandtschaft des Menschen mit dem Affen bewiesen.

Es ist höchst betrüblich, dass diese ungemein wichtigen Entdeckungen in dieser Weise missbraucht werden. Für die 
Begründung der Deszendenztheorie liefern sie ja nichts prinzipiell neues. Dass die systematisch einander am nächisten stehenden Tiere die zahlreichsten und weitgehendsten Uebereinstimmungen zeigen, ist eine selbstverständliche Tatsache, denn auf Grund der Uebereinstimmungen hat man ja die Systematik aufgebaut, indem man diejenigen Organismen, welche die grösste Uebereinstimmung zeigen, mit einander systematisch vereinigte. Im System stehen also diejenigen Lebewesen einander am nächsten, welche die grössten Uebereinstimmungen zeigen, und dass uns immer mehr solcher Uebereinstimmungen bekannt werden, je genauer die weiterschreitende Forschung die einzelnen Organismen kennen lernt, das ist auch keine neue Erfahrung. Es wäre wunderbar, wenn es anders wäre. Dass die systematischen. Uebereinstimmungen sich nicht nur auf morphologische, sondern auch auf physiologische Verhältnisse beziehen, entspricht allem bisher Bekannten. Dass das Blut in diesem Punkte eine Ausnahme machen werde, war nicht zu erwarten. Man würde wohl schon längst auf Grund der Tatsache, dass die roten Blutkörperchen der Säugetiere kernlos sind, im Gegensatz zu denjenigen der andern Wirbeltiere, die Blutsverwandt$s c h$ aft der Säugetiere unter einander für bewiesen erklärt haben, wenn man in jener Zeit, als diese Entdeckung gemacht wurde, schon so geistreich gewesen wäre wie heute. Die menschenähnlichen Affen sind eben diejenigen Tiere, die dem Menschen in allen Punkten am ähnlichsten sind - daher der Name. Kann es uns auffallen, wenn auch das Blut ähnlich ist? Neue Folgerungen können hieraus nur gezogen werden mittelst einer ganz unehrlichen oder ganz schwachsinnigen Vertauschung der Begriffe „Aehnlichkeit des Blutes“ und „Blutsverwandtschaft". Die Tatsache dieser „Blutsverwandtschaft" ist zwar hochinteressant und praktisch ungemein wichtig, für die Begründung der Deszendenztheorie hat sie keine grössere Bedeutung als jede andere Tatsache der vergleichenden Biologie.

Man hört so oft zur Begründung der Abstammungstheorie sagen, keine andere Theorie mache uns das Vorhandensein der vielgestaltigen und doch so viele übereinstimmenden Züge tragenden Organismenwelt verständlich. Wir müssen das zugeben, aber wir müssen hinzufügen, dass eine Entwicklungslehre, die uns über die Möglichkeit einer solchen Entwicklung nicht den geringsten Aufschluss gibt, uns die Sache eben auch nicht ver- 
ständlich macht. Und wenn gesagt wird, es sei doch unmöglich, sich vorzustellen, wie z. B. ein Elefant auf einmal entstand, so muss diesem unbestreitbaren Satze nur noch hinzugefügt werden, dass wir uns auch nicht vorstellen können, wie ein ganz einfacher Organismus, z. B. eine Amöbe, auf einmal entstanden sein kann. Dic Entstehung der Amöbe sich vorzustellen, wird nur deshalb von manchem für leichter gehalten, weil sie kleiner und einfacher gebaut ist. Aber dadurch wird das Rätsel nicht kleiner und einfacher. Die Entstehung der Amöbe und die Entstehung des Elefanten sich vorzustellen, ist gleich schwer, weil uns eben für beides jede Vorstellung fehlt. Wenn uns aber die Abstammungslehre über die erste Entstehung des einfachsten Organismus und über die Möglichkeit, wie aus ihm ein höherer sich entwickeln kann, heute noch keinen Aufschluss zu geben imstande ist, so ist die Frage wohlberechtigt, worauf denn eigentlich der Wert einer solchen Hypothese beruht. Die Vertröstung auf künftige Zeiten ist keine wissenschaftliche Grundlage, und ein Wechsel auf die Zukunft wird auch in der Wissenschaft erst in der Zukunft honoriert.

Verehrte Anwesende! Sie werden aus dem bisherigen Gedankengange meines Vortrages schon entnommen haben, dass ich ein überzeugter Anhänger der Abstammungslehre bin. Denn Sie werden mit mir der Meinung sein, dass gerade der Anhänger einer Lehre die Pflicht hat, alle Schwächen seiner Position rückhaltlos aufzudecken, und dem Gegner in allem Recht zu geben, worin er Recht hat. Und der Gegner könnte uns nicht ohne Grund den Vorwurf der Unehrlichkeit machen, wenn wir bestreiten würden, dass in diesem Punkte tatsächlich eine grosse Schwierigkeit für die Begründung der Abstammungslehre liegt, eine Schwierigkeit, über welche meistens nur deshalb allzuleicht hinweggegangen wird, weil ja, wie wir sahen, ursprünglich die Selektionstheorie es gewesen war, die uns von der Richtigkeit der Abstammungslehre überzeugt hatte. Haben wir bereits die Ueberzeugung von der Richtigkeit dieser Lehre, ist sie bereits auf andere Weise begründet worden, dann fügen sich die Tatsachen der vergleichenden Biologie sehr schön in dieselbe ein und helfen sie tragen. Ob aber diese Tatsachen allein genügen, um die Abstammungslehre zu begründen, diese Frage bedarf noch ëiner genaueren Prüfung. 
Hypothesen sind ja in der Wissenschaft etwas durchaus Berechtigtes und Notwendiges. Aber eine Hypothese ist nur dann berechtigt, wenn sie uns wirklich etwas leistet. Als von der Physik die Undulationstheorie des Lichtes aufgestellt wurde, lag ihre Berechtigung darin begründet, dass sie die Erscheinungen des Lichtes auf Bewegungsvorgänge zurückführen konnte, dass sie Phänomene, die vorher isoliert dastanden, in unsere sonstigen Vorstellungen eingliedern konnte, dass sie die Uebertragung der Gesetze der Mechanik, die sonst das Gebiet der Physik zu beherrschen schienen, auch für die Erscheinungen des Lichtes ermöglichte, welche vorher auf diese Gesetze nicht hatten zurückgeführt werden können.

Leistet die Hypothese der Abstammungslehre etwas ähnliches, wenn wir aus den biologischen Uebereinstimmungen zwischen einzelnen Organismenarten auf eine wirkliche Verwandtschaft schliessen?

Stehen die Tatsachen der vergleichenden Biologie in einem Widerspruch mit unseren sonstigen Erfahrungen über das Organische, der aufgelöst wird durch die Hypothese der Abstammungslehre? Kennen wir eine das Reich des Lebens beherrschende Gesetzlichkeit, welche auf die Tatsachen der vergleichenden Biologie nur angewendet werden könnte unter Voraussetzung der Hypothese der Abstammungslehre?

Die Beantwortung dieser Frage muss über die Berechtigung der Hypothese der Abstammungslehre entscheiden. Nur wenn sie das leistet, kann sie wirklich etwas Unverstandenes verständlich machen und für unsere Erkenntnis einen Fortschritt bedeuten.

Die Frage ist zu bejahen. Wir kennen eine solche Gesetzlichkeit, ja, es ist die einzige Gesetzlichkeit, die nach unserer Erfahrung das Leben beherrscht: das Gesetz, der Zweckmässigkeit. Mit diesem Gesetze der Zweckmässigkeit stehen eine Reihe von Tatsachen der vergleichenden Biologie wirklich in einem Widerspruch, der aufgelöst werden kann durch die Annahme der Abstammungslehre. Vor allem einer bestimmten Tatsachengruppe gegenüber scheint die Hypothese der Abstammungslehre dasjenige zu leisten, was ihr eine, auch der strengsten Prüfung standhaltende, wissenschaftliche Existenzberechtigung verleiht. Es sind das die sogenannten rudimentären Organe. Diese Erscheinungen werden allerdings schon längst mit Recht zur Be- 
gründung der Hypothese der Abstammungslehre herangezogen, wenn gleich man sich meistens nicht klar macht, welcher Standpunkt es ist, von welchem aus hier die Abstammungslehre ihre Begründung findet.

Wir können nicht leugnen, dass die rudimentären Organe einen Widerspruch bilden mit dem Prinzip der organischen Zweckmässigkeit, welches wir als die, das Organismenreich beherrschende, Gesetzlichkeit erkannt haben. Die verkümmerten Augen im Dunkeln lebender Tiere, z. B. des Maulwurfs, die kein Sehen ermöglichen, höchstens zu Entzündungen Anlass geben können, stehen im Widerspruch mit der sonst das Reich des Lebens beherrschenden Zweckmässigkeit. Das gleiche gilt von den Zähnen, die bei Walen während der Embryonalzeit auftreten, und mit denen die Tiere niemals beissen können, von den Flügeln der Strausse, mit denen diese Vögel nicht fliegen können, kurz, von den zahlreichen rudimentären Organen, die nicht funktionieren und dem Organismus nichts nützen. Sie scheinen gegen das Gesetz der Zweckmässigkeit zu sprechen und bilden dadurch einen Widerspruch mit der sonst sich uns aufdrängenden teleologischen Auffassung der organischen Natur. Dieser Widerspruch mit der teleologischen Auffassung wird beseitigt durch Einführung der Hypothese der Abstammungslehre.

Die Abstammungslehre liefert für die rudimentären Organe die Erklärung, dass dieselben als Stadien einer noch nicht abgeschlossenen Entwicklung im Sinne einer Rückbildung aufzufassen sind, die nötig geworden war durch eine veränderte Lebensweise der betreffenden Organismen. Wenn zum Beispiel eine bestimmte Tierart durch irgendwelche äusseren Umstände gezwungen wurde, in dunkeln Höhlen zu leben, so konnten ihnen ihre Augen nichts mehr nützen, sondern nur schaden, da die Augen ja leicht verletzliche Körperteile sind, und unter den jetzigen Lebensbedingungen um so eher Verletzungen ausgesetzt sind, als eben die dem Auge drohenden Gefahren durch seine Funktiori nicht mehr wahrgenommen wurden. Die Hypothese der Deszendenztheorie nimmt an, dass in solchen Fällen die zwecklos gewordenen Augen verschwinden durch einen allmählich im Lauf zahlreicher Generationen sich vollziehenden Rückbildungsprozess, und dass wir in den verkümmerten oder rudimentären Organen Gebilde zu erblicken haben, die sich in einem derartigen zweckmässigen Rückbildungsprozess befinden, der, 
allerdings aus völlig unbekannten Gründen, nur ganz langsam sich vollziehen kann, und deshalb zur Folge haben muss, dass manche Organismen - vielleicht alle - Generationen hindurch solche nicht mehr funktionierenden Organe in den verschiedensten Graden der Rückbildung mit sich schleppen müssen.

Nicht nur da, wo es sich um rudimentäre, also um solche Organe handelt, die gar nicht mehr funktionieren, zeigt sich unserer Betrachtung zuweilen ein Widerspruch mit dem Gesetz der Zweckmässigkeit. Die Untersuchung der verschiedenen Organismen lehrt uns eine grosse Anzahl von Einrichtungen kennen, dic zwar funktionieren, die aber in ihrer Anordnung, in der speziellen Ausführung, oft einen unnötigen Aufwand, eine unpraktische Komplikation, eine seltsame Umständlichkeit zeigen, so dass der Eindruck des Unzweckmässigen für uns hervorgerufen wird. So eingehende Beachtung man diesen Dingen schon längst geschenkt und so grossen Wert man solchen „dysteleologischen“ Einrichtungen für die Begründung der Deszendenztheorie beigelegt hat, so wenig hat man sich klar gemacht, dass ein Widersprich nur dann besteht, wenn das Gesetz der Zweckmässigkeit für das Organische vorausgesetzt wird. Denn wenn ein solches Gesetz das Organische nicht beherrscht, wenn das Lebende nur das Produkt eines blinden Zufalls ist, so kanı in dem Vorhandensein unzweckmässiger Einrichtungen nichts Auffallendes und kein Widerspruch liegen, der winer Erklärung bedürftig wäre. Nur die Voraussetzung der Teleologie erzeugt hier einen Widerspruch, nur sie ist es, die uns eine Erklärung fordern und finden lässt in der Abstammungslehre, die den Zustand zurückführt auf frühere Generationen, in welchen die Einrichtung zweckmässig war.

Mit der Einführung der Deszendenztheorie wird also die Durchführbarkeit teleologischer Auffassung erst ermöglicht für scheinbar zwecklose und unzweckmässige Einrichtungen, d. h. für Erscheinungen, die sonst der Einordnung unter das teleologische Prinzip widerstrebten, und die Hypothese setzt uns in den Stand, eine unseren sonstigen Erfahrungen entsprechende Gesetzlichkeit auch da durchzuführen, wo ohne diese Hypothese eine klaffende Lücke bestände.

Hier leistet uns also die Hypothese der Abstammungslehre etwas ganz anderes, als bei den früher besprochenen Tatsachen. der vergleichenden Biologie. Dass der Flügel des Vogels aus 
denselben Knochen aufgebaut ist, wie meine Hand, ist noch keine Tatsache, die im Widerspruch mit sonstigen Erfahrungen steht, und die mit ihnen in Einklang gebracht wird durch die Hypothese der Abstammungslehre. Hier hebt die Entwicklungslehrc keinen Widerspruch auf, sie löst kein Rätsel, sondern sie umschreibt ein Rätsel durch ein anderes Rätsel. Anders bei den von uns besprochenen Fällen. Wohl bringt auch hier die eingeführte Hypothese ein neues Rätsel mit, weil uns ja über die Art der Entwicklungsmöglichkeit jede Vorstellung abgeht. Aber die Hypothese beseitigt einen Widerspruch, der einer einheitlichen. Auffassung vorher entgegenstand.

Verehrte Anwesende! Das Ergebnis, zu welchem unsere Ueberlegungen geführt $\mathrm{zu}$ haben scheinen, ist ein recht merkwürdiges. Wir haben früher gesehen, dass die sogenannte moderne Biologie einen Gegensatz geschaffen hat zwischen Teleologie und Abstammungslehre, derart, dass die Abstammungslehre die Teleologie vernichtet habe. Unsere heutige Untersuchung scheint uns etwas anderes zu lehren. Die Abstammungslehre gibt nicht der Teleologie den Tod, sondern die Teleologie gibt der Abstammungslehre das Leben. Nur vom Standpunkt der Zweckmässigkeitslehre ist die Deszendenztheorie eine wissenschaftlich begründete Hypothese, mit anderen Worten: die Teleologie ist die einzige Begründung der Abstammungslehre. 
II.

Der Lamarckismus wurde bisher als eine Theorie aufgefasst, welche eine Erklärung für die Tatsache der organischen Zweckmässigkeit geben soll. Indem der Lamarckist die Zweckmässigkeit eines Organs als Produkt einer durch viele Generationen fortgesetzten Funktion ansah, glaubte und glaubt er die Entstehung des betreffenden Organs „mechanisch" d. h. unter Ausschluss teleologischer Prinzipien erklärt zu haben. In Wirklichkeit ist das ja nicht der Fall. ${ }^{1}$ ) Der Lamarckismus leidet an dem nämlichen Grundfehler, wie der Darwinismus. Er setzt Organismen voraus, also Körper, die durch gar nichts anderes charakterisiert werden können als durch die Eigenschaft, zweckmässiger Reaktionen fähig zu sein.

Neben dieser ganz allgemeinen Voraussetzung dessen, was er erklären soll, enthält der Lamarckismus im Speziellen ein Prinzip von teleologischem Charakter. Denn die Fähigkeit, durch Uebung zu gewinnen, das Fundament des Lamarckismus, ist eine im höchsten Grade zweckmässige Einrichtung, die also unfähig ist, eine Theorie zu begründen, welche für die organische Zweckmässigkeit eine Erklärung liefern soll. Jeder Lamarckist ist demnach Teleologe ohne es $\mathrm{zu}$ wollen und ohne es zu wissen, und der teleologische Charakter der Lehre kam und kommt ihren Anhängern so wenig zum Bewusstsein, dass der Lamarckismus genau in der nämlichen Weise als antiteleologische Theorie verwendet wird wie die Zuchtwahllehre von den Darwinianern.

Ob Lamarck selbst des teleologischen Charakters seiner Lehre sich bewusst war, ist eine mehr untergeordnete Frage von nur historischem Interesse. Es wird diese Frage neuerdings entschieden bejaht von August Pauly, der vor zwei Jahren seine durchaus teleologischen Anschauungen über das Organische ver-

1) vgl. meine „Beiträge zur Kritik des Darwinismus,“ Leipzig 1898 p. 63. 
öffentlicht hat, die er als Lamarckismus bezeichnet. ${ }^{1}$ ) Schon hieraus ist ersichtlich, dass Pauly unter Lamarckismus etwas anderes versteht, als was bisher darunter verstanden wurde.

So sehr ich mit Pauly darin einverstanden bin, dass ein teleologisch zu beurteilender Faktor die Umwandlung der Organismen hervorgerufen haben muss, so wenig kann ich ihm zustimmen bezüglich des speziellen Charakters, den Pauly diesem Faktor zuerteilt. Zunächst halte ich es, wie ich dies früher ${ }^{2}$ ) schon dargelegt habe, für prinzipiell unrichtig und wissenschaftlich völlig wertlos, das als teleologisch anerkannte Prinzip deshalb, weil wir etwas Analoges nur aus unserem eigenen Seelenleben kennen, ohne weiteres mit dem Psychischen zu identifizieren. Zweitens aber glaube ich, dass Pauly, der völlig im Recht ist, wenn er dem lamarckistischen Prinzip einen teleologischen Charakter zuspricht, doch völlig im Unrecht ist, wenn er dem, das Organismenreich beherrschenden, teleologischen Prinzip einen lamarckistischen Charakter gibt. Denn er verleiht dadurch dem teleologischen Prinzip eine Beschränktheit, die es nachweislich nicht hat.

Lamarckistisch ist Pauly's Auffassung insofern, als sie von dem Grundsatz beherrscht wird: zuerst die Funktion, dann das Organ. Dieser lamarckistische Grundsatz, welcher nur deshalb nicht paradox ist, weil er die dem Lamarckismus ebenso wie dem Darwinismus anhaftende Beschränktheit ausspricht, nur mit Fortsetzungen arbeiten zu können, ist auch die Grundlage der Pauly'schen Auffassung. Im Gegensatz zu Darwin, bei welchem „die Variante in einem andern Zeitpunkt erscheint als derjenige ist, in welchem ihre Grösse und ihr Charakter allein bestimmt werden kann“, nämlich dem „Augenblick der Funktion, der tatsächlichen Verwendung der Veränderung", rühmt er Lamarck, welcher als Einziger die Tatsache nicht übersehen habe, „dass der Zeitpunkt der Entstehung eines funktionellen Zweckmässigen mit dem Zeitpunkt der Ausübung der Funktion zusammenfallen, eine Wirkung oder Nachwirkung der Funktion sein muss, weil nur in der Funktion die fördernde Wirkung einer eingetretenen Veränderung verspürt werden kann, in jedem anderen Fall aber

1) August Pauly, Darwinismus und Lamarckismus Müunchen 1905.

2) Mechanismus u. Vitalismus. II. Auflage, p. 13 u. ff. 
die einzelnen neuen Beiträge zu einer oft sehr komplexen Funktion irgendwie vorausbestimmt worden sein müssten." ${ }^{\text {) }}$ )

Diese grundlegenden Voraussetzungen der Paulyschen Auffassung werden aber nicht näher begründet, sondern als selbstverständlich angenommen. Denn es ist keine Begründung, wenn Pauly sagt, ,die Unmöglichkeit, das Organ früher als die Funktion auftreten zu lassen, leuchtet jedem ein" ${ }^{\prime 2}$ )

Die Richtigkeit dieses Satzes ist keineswegs unmittelbar einleuchtend. Zunächst ist im Gegenteil nur das Eine unmitteltar einleuchtend, dass jede Handlung ein handelndes Subjekt, jede Funktion ein funktionierendes Organ voraussetzt, und dass nur die Annahme einer fortgesetzten Funktionssteigerung oder eines Funktionswechsels jenen Satz davor bewahren kann, eines Sinnes zu entbehren. Wenn das Bein als Flosse benützt wird, so war in gewissem Sinne die Flosse noch nicht da, bevor sie als solche zur Verwendung kam, sondern das Bein wurde zur Flosse dadurch, dass es als solches benutzt wurde, und die weitere Benutzung schuf nun - ich rede ex hypothesi - das Organ imımer weiter zur Flosse um.

Aber ein solcher Prozess darf keineswegs als selbstverständlich angesehen werden, weil es zahlreiche Fälle gibt, für welche dieser Vorgang lauch dann unvorstellbar ist, wenn man, wie Pauly dies tut, den Lamarckismus dahin ergänzt, dass man an Stelle der direkten Wirkung der Funktion das ,Innewerden der Wirkung (63) setzt. Abgesehen davon, dass diese psychologisierende Umgestaltung des Lamarckismus den Boden der Tatsachen vollständig verlässt und eine willkürliche Interpretation gibt, über deren Richtigkeit oder Unrichtigkeit gar nicht diskutiert werden kann, sondern von der höchstens der Einzelne anzugeben vermag, ob sie seinem Geschmack zusagt oder nicht, - abgesehen davon ist diese Interpretation nicht imstande, den Machtbezirk des Lamarckismus wirklich zu erweitern und diejenigen Fälle in sein Bereich żu ziehen, denen gegenüber er bisher mit Recht als unanwendbar erkannt worden ist.

Wenn Pauly sagt, ${ }^{4}$ ) es sei falsch, dass das Organ früher sei als seine Funktion, weil man sonst als organbildende Ursache ,eine vorausdenkende Macht statuieren würde, welche

\footnotetext{
1) pag. 34 .

2) pag. 49 .

3) pag. 17 .

4) pag. 48 .
} 
Organe erzeugt, bevor noch der Besitzer derselben ein Bedürfnis für sie empfindet", so läge in dieser Ausführung nur dann ein zwingendes Argument, wenn nachgewiesen wäre, dass eine derart vorauswirkende Kraft völlig ausgeschlossen ist. Dies wird von Pauly als selbstverständlich vorausgesetzt, ein Nachweis wird gar nicht versucht, der Versuch müsste aber in jedem Falle misslingen aus dem einfachsten Grunde, weil im Gegenteil gerade gezeigt werden kann, dass es zahlreiche Beispiele gibt, aus denen mit Sicherheit zu ersehen ist, dass auch im Pauly'schen Sinne die Funktion keinen Einfluss auf die Vervollkommnung eines Organes oder einer Einrichtung hat ausüben können.

Schon die Fälle von Mimikry werden lamarckisch nicht verständlicher durch die Einführung der Pauly'schen Interpretation. Wie kann überhaupt der Faktor des „Innewerdens der Wirkung der Funktion“, der „Empfindung des Bedürfnisses“, des „Urteils", - wenn wir, was Pauly ja will, diesen psychologischen Ausdrücken mehr als die Bedeutung einer bildlichen Umschreibung beilegen wollen - auf die Fälle von Mimikry Anwendung finden? Hat das Hermelin das Bedürfnis empfunden, weiss zu werden, und hat die Empfindung dieses Bedürfnisses es weiss werden lassen? Und wie sollen wir uns vorstellen, dass hier die beginnende Wirkung dieser „Funktion" behufs weiterer Vervollkommnung wahrgenommen werden konnte von dem Tier, welches das Resultat doch wohl höchstens an seinen Vorderpfoten wahrnehmen konnte?

Pauly versucht zwar einen Weg anzudeuten, auf welchem auch die Erscheinungen der Mimikry seiner Erklärung zugänglich werden. Er stellt den Satz auf, dass „Seelenzustände jeder Art teleologische Wirkungen ausüben" ${ }^{\prime 1}$ ) können. Dieser Satz wird allerdings von Pauly in keiner Weise bewiesen, sondern es werden nur Beispiele zweckmässiger Anpassung der äusseren Gestalt an vorhandene Bedürfnisse angeführt und nur die Behauptung hinzugefügt, dass sich solche Verhältnissennach Empfindungen gebildet haben ,müssen“ $\left.{ }^{\circ}{ }^{2}\right)$ Und mit Hülfe eines derart begründeten Satzes gelangt Pauly zu folgenden Ableitungen:

„Wenn wir Seelenzustände jeder Art teleologische Wirkungen ausüben sehen, so ist es eine notwendige Konsequenz, dass einer der häufigsten Zustände, die Angst um das eigene

1) pag. 242 .

) pag. 241 . 
Leben, die sich in dem Benehmen so vieler Tiere ausdrückt, nicht ohne Wirkung bleiben kann auf Farbe und Form des Körpers, wobei die Wirkung keineswegs eine lebensrettende zu sein braucht, sondern nur eine natürliche Folge des seelischen Zustandes und der Mittel ist, welche dem Bedürfnis, ungesehen zu bleiben, entsprechen.

Dieses Bedürfnis kommt auch dem Raubtier zu, und demgemäss sehen wir es auch oft in seiner farbigen Erscheinung oder in seiner Zeichnung mit der Farbe oder dem Eindruck seiner Umgebung übereinstimmen, ohne dass diese Uebereinstimmung als eine Bedingung seiner Existenz angesehen werden könnte." 1)

Zunächst' ist zu bemerken, dass bei vielen Organismen, und gerade bei Raubtieren, die Aehnlichkeit mit der Umgebung nicht dazu dient, von den gefürchteten Feinden, sondern von den keineswegs gefürchteten Beutetieren nicht gesehen zu werden, wobei also die „Angst um das eigene Leben" nur in sehr indirekter Weise, nämlich als Angst um das eigene Futter, zum Ausdruck kommen könnte. Ausserdem sind bekanntlich auch häufig dic Eier eines Tieres durch eine der Umgebung entsprechende. Färbung geschützt, und zwar nicht nur bei brütenden Vögeln, die sich um ihre Eier kümmern, und von denen eventuell noch behauptet werden könnte, sie machten Erfahrungen über das Schicksal der Eier, und die hierbei empfundene Angst könne dann die Färbung der späteren Eier veranlassen, sondern auch bei Tieren, die sich gar nicht um die gelegten Eier kümmern, z. B. Insekten. Wer hat die Angst, das Ei oder die Mutter?

Vor allem aber ist es völlig unverständlich, wie ein Angstgefühl den Körper seiner Umgebung ähnlich machen soll, wie dieses Gefühl Aehnlichkeiten mit Verhältnissen hervorzurufen vermag, von denen das ängstliche Tier gar nichts wissen kann, wie dieser Affekt insbesondere Aehnlichkeiten hervorrufen kann nicht nur mit dem Gesamteindruck der Umgebung, sondern mit isolierten Erscheinungen, z. B. mit einem dürren Blatt oder einem andern Tier. Hier müsste denn doch eine etwas allzusehr spezialisierte Angst vorausgesetzt werden.

Es wird unserer Vorstellungskraft hier ebensoviel zugemutet, wie bei der Erklärung, die Pauly für die Entstehung des schönen Gefieders mancher Vögel zu geben versucht. Auch

1) pag. 242. 
hier wird eine Empfindung für den wirkenden Faktor erklärt, ohne dass sich die Pauly'schen Ableitungen über den Charakter einer blossen Behauptung erheben. Ich wenigstens bin nicht imstande, eine Begründung zu erblicken in Sätzen wie den folgenden:

„Den merkwürdigsten Einfluss aber von allen, denen sie nachgibt, erfährt die Feder in der Bestimmung ihrer farbigen Erscheinung, in welchem sich geradezu die Wirkung von Vorstellungen offenbart, welche aus Sinneswahrnehmungen hervorgegangen sein müssen, bei denen die Ursache ihren Sitz nur im Gehirn gehabt haben konnte und die Wirkung nur ihre äusserste Oberfläche der Feder betraf.

Der erste Eindruck, welchen die Farbenpracht eines Pfaues hervorruft, ist der, dass das gestaltende Prinzip, welches seine Farbenerscheinung beherrscht, ein optisches gewesen sein muss. Die Ursache muss Augen gehabt haben. Sie muss eine ähnliche Freude an Farbe und Glanz gehabt haben, wie wir selbst. Das Tier selbst sagt es uns, durch den Stolz, mit dem esi sein schönes Gewand trägt, durch die aus seinem Innern kommende Muskelwirkung, auf die Federn, durch die es sie in einem Rad vor uns ausbreitet.

Es verrät uns seinen ursächlichen Anteil an seiner Pracht, so gut wie die unscheinbar gefärbten Vögel durch die Furcht vor ihren Feinden ihren ursächlichen Ariteil an der schützenden Farbe ihres Gefieders verraten, welche mit der Farbe der Umgebung übereinstimmt und mit ihr je nach der kalten oder warmen Jahreszeit wechseln kann." ${ }^{1}$ )

Da wir uns gar nicht vorzustellen vermögen, wie eine Empfindung solche Umgestaltung hervorrufen kann, und da ferner solche etwaigen Empfindungen des Pfaues, der seine Schwanzfedern und sein Rad so gut wie gar nicht sieht, völlig ausser Stande sein müssten, die Umwandlung der Federn zu kontrollieren, - was können uns solche „Erklärungen“ helfen? Sie können uns nur zurückwerfen in die Spekulationen einer überwundenen Naturphilosophie.

Den Fällen von Mimikry gegenüber muss also der Lamarckismus in jeder Gestalt versagen. Aber es gibt noch genug andere Beispiele, in denen das vom Lamarckismus geforderte zeitliche Verhältnis zwischen dem Auftreten eines Organes und

1) pag. 271 u. ff. 
dessen Verwendung nicht zutreffen kann. Besonders schwierig wird diese Lamarckistische Annahme für alle diejenigen Fälle, bei welchen nicht am unmittelbaren Ort des Bedürfnisses, sondern an einer entfernteren Stelle für die Befriedigung des Bedürfnisses gesorgt werden muss. Pauly hat diese Schwierigkeit wohl erkannt, und er ist ihr nicht aus dem Weg gegangen, sondern er hat diesem Verhältnis eine eingehende Besprechung gewidmet. Er hilft sich in solchen Fällen mit der Annahme einer „Association“, einer „Ausstrahlung“. „Der teleologische Vorgang als eine Association zwischen Bedürfnis und der Erfahrung eines Mittels hat infolge dieses Verhältnisses Ausdehnung. Diese ist von der Grösse der Entfernung der beiden Punkte abhängig, an welchen das Bedürfnis und àn welchen das wirkende Mittel liegt. In organischen Körpern von grossen Massen kann diese Entfernung sehr beträchtlich sein, sie wird aber um so kleiner, je weiter zurück wir den Vorgang nach der Richtung seines phylogenetischen Ursprungs verfolgen, und wir können mit unserer Vorstellung nach dieser Seite keine Grenze erreichen. Zwischen der Wurzelspitze eines Baumes und einem Blatt seiner Krone muss der Austausch der Zuständlichkeit ihrer Zellen ebenso sicher möglich sein, als zwischen den Protoplasmamolekülen im Innern eines einzelligen Wesens und den Molekülen der äussersten Körpergrenze desselben.

Diese Möglichkeit, die inneren Zustände verschiedener Punkte vermittelst Leitung auszutauschen, ist die oberste dynamische Voraussetzung aller organischen Teleologie. Ohne sie wäre weder der Aufbau zusammengesetzter, aus vielen Zellen bestehender lebendiger Körper möglich, noch die Existenz einzelliger zweckmässig organisierter und zweckmässig reagierender Wesen, weil ohne sie weder Intellekt als aktive Synthesé noch zusammengesetzte Empfindungszustände als dessen Ursache und Folge möglich wären. Denn die Verknüpfung verschiedenartiger Empfindungszustände in einem Subjekt ist die Bedingung allen Urteils und Urteil die Bedingung aller Teleologie." ${ }^{\text {(1) }}$

Im einzelnen sucht Pauly dieses Verhältnis z. B. klar zu machen an der zweckmässigen Art, wie rankende Kletterpflanzen die nötige Festigkeit gewinnen. Pauly sagt hierüber: „Mag nun das Haftorgan sich an die Fläche einer Wand ansetzen oder,

1). pag 163 . 
wie zuweilen beim wilden Wein, durch Eindringen der Ranke in einen Spalt in diesem seinen Halt finden, immer kann eine genügende Befestigung derselben an ihrer Unterlage nur dadurch erfolgen, dass sich die Zellteilungen in dem Haftorgan nach der Form der von ihr berührten Fläche oder des von ihr erreichten Spaltes richten. Die sich anschmiegende Fläche des Haftorgans muss den Zufälligkeiten der Form der Unterlage, die sie berührt, mit ihren Zellteilungen folgen, und diese müssen dabei so geleite1 werden, dass sie durch Ausnützung der konkreten Verhältnisse eine dem Zug an der Ranke möglichst Widerstand leistende Befestigung erzielen. Dies setzt sowohl Berührungsempfindung als auch die Empfindung des erreichten Widerstandes gegen Zug voraus, und da Ranke und Haftorgan nicht für sich selbst, sondern im Dienste der Pflanze handeln, deren Bedürfnis nach Stütze sie erfüllen, so setzt ihre Tätigkeit eine korrespondierende Leitung zwischen dem Sitz des Bedürfnisses nach Stütze und den Organen, welche dasselbe erfüllen können, voraus, eine Korrespondenz, welche sich nïcht allein zwischen den unmittelbar beteiligten Organen, dem Stengel und der Ranke mit ihren Haftorganen abspielt, sondern nach früher Gesagtem auf alle Zellen der Pflanzen ausstrahlen muss. Hier muss die Regulierung der Zellteilung und damit das Urteil viel komplizierter sein, als bei den Bewegungen der Schlingpflanzen und der Ranken; denn wenn auch für eine in einen Spaltraum gedrungene Ranke ein ungeregeltes Wachstum des Haftknöpfchens zu einer festen Einkeilung genügen würde, so würde doch ein solches regelloses Wachstum bei einem saugnapfartig auf einer Fläche aufliegenden Haftorgan keine Befestigung erzielen. Bei diesem muss den Vorsprüngen der Unterlage gegenüber die Zellteilung gehemmt, den Hohlräumen gegenüber angeregt werden, und ausserdem das Wachstum im Sinne einer Umklammerung sowie Einkeilung geleitet werden.

Bei diesen Korrespondenzen zwischen weit von einander entfernten Pflanzenteilen spielen sich associative Vorgänge $a b$, welche nichts anderes sind als Denkakte der Pflanze. Ihr primäres Bedürfnis nach Licht und Luft, welches infolge der ungenügenden Tragfähigkeit des Stengels das sekundäre Bedürfnis nach Stütze hervorrief, muss, bei seiner allseitigen Âusbreitung im ganzen Körper der Pflanze, in denjenigen Organen, welche bei ihrem Wachstum mit festen Körpern in Berührung zu 
kommen Gelegenheit hatten, die zweckentsprechenden Bewegungen hervorgerufen haben. Dem früher Gesagten zufolge muss die vom primären Ort des Bedürfnisses ausgegangene Erregung am Orte der Befriedigungsmittel einen rückläufigen Strom der Erregung, welcher als Inhalt Gefühl und Vorstellung des Befriedigungsvorganges enthält, bewirken. Bedürfnis und Befriedigung bilden einen Stromkreis, dessen Bahn während des Vorgangs kontinuierlich durchlaufen wird mit wechselnder Stärke der Erregung, die von dem Grade des Gelingens der Befriedigung abhängig ist." ${ }^{* 1}$ )

Selbst wenn wir zugeben könnten, dass für das vorliegende Beispiel durch die wiedergegebenen Darlegungen unserm Bedürfnis nach Verständnis die Mittel zur Befriedigung geboten worden seien, so müssten wir dies aufs bestimmteste verneinen für alle diejenigen Vorgänge, bei denen ein Körperbestandteil erst funktionieren und von einem anderweitigen Bedürfnis, das er befriedigen soll, erst Kenntnis erhalten könnte zu einer Zeit, da er selbst den Ort, an dem er entsteht und etwaiger Aenderungen noch fähig wäre, verlassen hat. Ein solches Beispiel sind z. B. die Blutkörperchen. Sie entstehen an bestimmten, im Organismus verteilten Bildungsstätten, die sie, sobald sie fertig sind, verlassen, um im Körper umherzuwandern und erst zu funktionieren, nachdem sie nicht mehr in der Lage sind, auf Grund von Erfahrungen sich umzubilden oder Nachkommen zu erzeugen, denen ihre Erfahrungen zu gute kommen könnten. Und wie sollte denn überhaupt ihnen über die Bédürfnisse, die sie in den verschiedensten Körperteilen zu befriedigen haben, eine Erfahrung zufliessen können, nachdem sie den organischen Zusammenhang mit dem Körper aufgegeben haben, dergestalt, dass auch eine Ausstrahlung im Pauly'schen Sinne keinen Einfluss auf sie und ihre Nachkommen (die es gar nicht gibt) ausüben könnte? Wie könnte diesem Beispiel gegenüber der Lamarck-Pauly'sche Grundgedanke, dass nur die Funktion es ist, die das Organ umändert, aufrecht erhalten werden, hier, wo das funktionierende Organ überhaupt keiner Aenderung, am allerwenigsten einer fortwirkenden Aenderung fähig ist?

Das Organ hat hier zur Zeit der Funktion den Körperverband eigentlich verlassen, und gerade hieraus resultiert am deutlichsten die Unmöglichkeit, etwaige Erfahrungen dem Körper

1) pag. 182 u. 183. 
zu gute kommen zu lassen. Es gibt Vorgänge, an denen dieses Verhältnis vielleicht noch deutlicher gezeigt werden kann. Betrachten wir z. B. die Fähigkeit des Blutes, zu gerinnen. Diese ungemein zweckmässige Einrichtung, die den Organismus davor schützt, bei Verletzungen zu verbluten, kommt erst zur Geltung, wenn das Blut den Körper verlassen hat. Das ausgetretene Blut gerinnt und verklebt die Wunde. Diese Gerinnung ist kein Lebensvorgang mehr, sie ist also keine Funktion, die in lamarckistischem Sinne ihre eigene Potenz steigern kann. Zuerst muss die Einrichtung da sein, dann kann es erst zur. Funktion kommen und die Funktion kann keinen Einfluss auf die Entstehung und Vervollkommnung der Einrichtung ausüben. Jene Bildungsstätten, in denen die bestimmten Blutbestandteile bereitet werden, welche die Gerinnungsfähigkeit herstellén, können in ihrer Arbeit nicht beeinflusst werden durch die mehr oder weniger häufige spätere Inanspruchnahme dieser Eigenschaft des Blutes.

In analoger Weise sind zu beurteilen alle diejenigen Sekrete, die nach der Ausscheidung zu funktionieren haben. Woher sollen die Magendrüsen erfahren, dass der ausgeschiedene Magensaft die Speisen verdaut hat? Der Verdauungsprozess, ein rein physikalisch-chemischer Vorgang kann keinen Einfluss auf die Vervollkommnung der Magendrüsenfunktion ausüben. Wollte man annehmen, dass die Magendrüsen infolge der mehr oder weniger vollständigen Resorption der Speisen und der daraus resultierenden mehr oder weniger vollkommenen Ernährung des Gesamtkörpers am eigenen Leibe spüren, ob sie ihre Sache recht gemacht haben, oder ob der Magensaft noch nach dieser oder jener Richtung einer Verbesserung bedarf, so würde sich die Erklärung so sehr ins Unkontrollierbare und Willkürliche verlieren, dass von einer wissenschaftlichen Diskussion keine Rede mehr sein könnte, und vollends unmöglich wäre ein derartiger Ausweg z. B. gegenüber dem Sekret der Muttermilch.

Woher sollen die Milchdrüsen erfahren, ob ihr Produkt dem Kinde gut bekommen ist? Wie kann die so ungemein zweckmässige und komplizierte Zusammensetzung der Muttermilch Folge ihrer „Funktion" sein, die in einem andern Organismus sich vollzieht und die nur darin besteht, von diesem andern Organismus aufgenommen zu werden? 
Gerade bei solchen Einrichtungen, die dem Wohl der kommenden Generation dienen, also bei den Einrichtungen der Brutpflege in weitesten Sinne, wird die Unanwendbarkeit des Lamarckismus auch in der Pauly'schen Form am deutlichsten.

Auf welche Weise erhielten die Eier der Vögel bezw. ihrer Vorfahren, der Reptilien, eine Kalkschale? Das Bedürfnis, welchem die Kalkschale begegnet, ist der Schutz gegen aussere Einwirkung. Dieses Bedürfnis kann der mütterliche Organismus, der die Kalkschale produziert, nicht empfunden haben, denn das Bedürfnis tritt erst auf, wenn das Ei gelegt ist. Ein Bedürfnis ist die Kalkschale für den sich entwickelnden Organismus; dessen Bedürfnis, selbst wenn er es empfinden sollte, kann aber keinen Einfluss auf die Beschaffenheit der Schale ausüben. Die Schale ist da, sie funktioniert, indem sie den jungen Organismus schützt, und dieser macht Gebrauch von ihrem Schutz. Aber selbst wenn man soweit gehen will, anzunehmen, dass er diesen Schutz als Wohltat empfindet, dass es also zu einem „Innewerden der Wirkung der Funktion“ kommt, ganz unmöglich ist es, dieses Innewerden der Funktion irgend einen Einfluss auf die Gestaltung der Schale gewinnen zu lassen. Dies könnte höchstens geschehen durch die Annahme, das junge Tier mache Beobachtungen über die Licht- und Schattenseiten der Kalkschale und verwerte seine Beobachtungen später im Interesse der folgenden Generation, eine Annahme, gegen welche allerdings nichts mehr gesagt werden könnte.

Eine ähnliche Ueberlegung wird uns sofort die Unmöglichkeit darlegen z. B. den Instinkt zum Nestbau im Pauly'schen Sinne zu erklären. Das Empfinden der Wohltat des warmen Nestes, kann auf den Instinkt zum Bau desselben keinen Einfluss ausüben. Denn wollte man selbst so weit gehen, - was aber eine wissenschaftlich wertlose Annahme wäre - vorauszusetzen, der Vogel erinnere sich beim Nestbau der Annehmlichkeiten, die er selbst seinerzeit in seinem ersten Neste empfunden, so könnte er sich doch höchstens erinnern an die Vorteile, die ihm das Nest gebracht, nicht aber an die Art der Herstellung, die er ja nicht miterlebt hat. Er macht also keine Erfahrungen, die ihn befähigen könnten, das Nest besser zu bauen als seine Eltern.

Die viel zitierten Bemerkungen, die Autenrieth über das Gespinnst des Nachtpfauenauges macht, haben die Pauly'sche 
Auffassung schon im voraus so treffend widerlegt, dass ich mich nicht enthalten kann, sie hierher zu setzen:

„Wenn die Raupe des Nachtpfauenauges, Phalaena saturnia, pavonia L., an das obere Ende ihrer Puppenhütte ein doppeltes Gewölbe von steifen, besonders an dem innern Gewölbe mit ihren Spitzen zusammentreffenden, freien, nur an der Spitze mit ganz feinen Fädchen vereinigten Borsten aus Seide spinnt, das auf leichten Druck von innen aus sich öffnet, von aussen auch bei starkem keinen Zugang ins Innere gewährt, so müssten sie, wenn sie dabei nach Ueberlegung oder Verstand handelte, folgendes, menschlicher Vorstellung nach, bedacht haben: dass sie in Puppenzustand geraten und darin jedem ungünstigen $\mathrm{Zu}$ fall ohne mögliches Entrinnen hingegeben sein würde, wenn sie nicht voraus für hinreichenden Schutz sorgen, dass sie aus ihrer Puppenhülle als Schmetterling werde hervorgehen sollen, ohne Organe oder Kräfte zu haben, die von ihr noch als Raupe gesponnene Hülle zu durchbrechen, oder ohne, wie andere Falter, einen Seidegespinnst auflösenden Saft von sich geben zu können, dass sie also, ohne schon als Raupe für einen schicklichen Ausgang aus ihrem Gehäuse gesorgt zu haben, yor der Zeit in demselben würde sterben müssen. Dagegen müsste ihr im Augenblicke ihres Geschäftes auch klar sein, dass sie, um einst als Schmetterling ihr Gespinnst verlassen zu können, nur ein Gewölbe mit der Eigenschaft zu verfertigen habe, von aussen herein zu schützen, von innen heraus aber mit leichter Mühe geöffnet zu werden, dass dieses geschehen werde, wenn sie es aus steifen in der Mitte sich zusammenneigenden, sonst freien Seidenborsten verfertige; und überdies müsste sie voraus wissen, dass sie dazu nur den Seidenstoff, mit welchem sie den übrigen Umfang ihrer Hülle erbaue, jedoch hier mit regelmässigerer Künstlichkeit anzuwenden habe. Und doch konnte sie in nichts von allem diesen von ihren Eltern unterrichtet worden sein, denn diese waren längst tot, als sie noch aus dem Ei schlüpfte. Sie konnte es auch nicht erst durch 'Uebung oder Erfahrung: erlernt haben, denn sie vollbringt ihr Kunstwerk in ihrem Leben nur einmal, auch nicht durch Nachahmung, denn sie lebt nicht gesellschaftlich. Ihr Verstand aber konnte in ihrem ganzen Raupenzustande nur äusserst wenig ausgebildet werden; sie kroch auf dem Gesträuche umher, auf welchem sie zuerst das Licht erblickt hatte, um seine Blätter zu fressen, wozu sie keines 
Nachdenkens bedurfte, weil diese sich ihr von selbst darboten; sie hielt sich etwa mit den Füssen an, um nicht zu Boden zu. fallen, und begab sich, um von dem Regen nicht nass zu werden, auf die Unterseite eines Blattes; sie entledigt sich zwar einige Male ihrer alt und unbequem' gewordenen Haut unter unwillkürlichen Krümmungen ihres ganzen Körpers, aber ohne damals sich einzuspinnen; dies war ihr ganzes Leben, ihre ganze Verstandesübung."

In analoger Weise suchte Autenrieth an den Instinkthandlungen der Mauerwespe ${ }^{2}$ ) die Annahme zu widerlegen, dass bei der Entstehung derartiger Instinkte Erfahrungen eine Rolle spielen.

„Betrachte man das bekannte Beispiel der Mauerwespe Réaumurs (entweder Epigone spinipes oder Odynerus murarius von Latreille). Nachdem dieses Insekt in festen Sand eine enge zylindrische Höhle zum Teil vier Zoll tief gegraben hat, legt es in den Boden von dieser ein $\mathrm{Ei}$, und schichtet nun ringförmig zusammengekrümmte, aber noch lebende grüne kleine ohnfüssige Maden fest darauf. Der übrige Teil der Höhle oder der in den Sand gegrabene Röhre wird von der Wespe wieder genau mit Sand zugefüllt. Die Maden, deren ungefähr ein Dutzend sind, bleiben leben, bis die aus dem Ei schlüpfende Wespenlarve eine nack der andern verzehrt hat. Réaumur glaubt, die Mutterwespe suche deswegen Maden aus, die alle von gleichem Alter, ihrer eigenen Verwandlung nahe, ohne Nahrung ungefähr 14 Tage lang beim Leben bleiben könnten. Seinen angestellten Versuchen nach scheint die Wespenlarve genau so viele Maden als Mitgabe in ihre Höhle zu erhalten, als sie braucht, um, wenn die letzte derselben von ihr verzehrt ist, in den Puppenzustand überzugehen, welchen sie dann erst im Frühjahr des folgenden Jahrs als selbst wieder vollkommene Maurerwespe verlässt.

Dupont de Némsurs sucht nun, ohne einen Instinkt anzunehmen, die Frage, woher die Mutterwespe wisse, wie viele Maden die künftige Larve bedürfe, um zu ihrer Vollkommenheit, zu gelangen, damiı zu beantworten, dass er behauptet, noch als Wespe erinnere sie sich genau, in welchem Zustand als Larve sie sich befunden habe; ihr Puppenzustand sei nur ein

1) Autenrieth, Ansichten über Natur und Seelenleben, Stuttgart und Augsburg 1836. pag. 171 u. ff.

2) l. c. p. 172 u. ft. 
Schlaf gewesen, aus welchem sie erwacht sei, wie wir auch erwachen, ohne das Andenken an das verloren zu haben, was wir vor unserem Schlafe erlebt haben. Genötigt nach ihrer Verwandlung, durch ëgene Kraft sich 'durch den die Röhre verstopfenden Sand zu arbeiten, erhalte sie auch von der Beschaffenheit dieser Höhle die genaueste Kenntnis. Sie wisse sonst von nichts; kaum dass in ihrem vollkommenen Zustand einige Augenblicke lang die Liebe sie beschäftige; sei 'der Taumel dieser aber vorüber, so kehre sie zu den süssen Erinnerungen aus ihrer Kindheit zurück. Wie unsere Kinder sich Häuschen bauen, so ahme sie nun selbst nach, was sie bereits als Werk anderer kennen gelernt habe, und im Bewusstsein, wie zärtlich ehmals für sie gesorgt worden sei, spiele sie nun auch die treue Sorgerin. Ihre Arbeit, der einzige Gegenstand ihrer eifrigen Unterhaltung, ermüde sie von Zeit zu Zeit, die Anstrengung treibe sie, da sie schwanger sei, zur Geburt, und sie lege ein $\mathrm{Ei}$, wahrscheinlich ohne $\mathrm{zu}$ wissen, was dieses sei. Das Werk ihres Eifers aber müsste vollkommen werden, sie suche also auch grüne Maden und finde sie. Sei nun aber alles ausgeführt, so sterbe sie, erschöpft von der Arbeit und jetzt aus Ueberdruss, wie andere Tiere auch.

Doch fand Dupont selbst die Frage von Cuvier etwas bedenklich: woher weiss denn die Maurerwespe, dass die Maden, welche sie als Larve frass, grün waren? Als die Maden da waren, konnte die Larve sie nicht sehen; und als die Wespe sah, waren alle Maden in der finsteren Höhle schon längst gefressen. Man könnte auch sonst noch ein und anderes fragen, z. B. da nach Réaumurs Beobachtung nicht alle Höhlen, deren dieses Insekt mehrere nacheinander gräbt, gleich tief sind, also nicht alle gleich viele Anstrengung erfordern, warum kommt es, weníi die Maurerwespe Eier legt, weil sie sich abmüht, dass nie die grünen Maden zuerst eingetragen werden, und das Ei zuletzt gelegt wird, sondern immer nur umgekehrt? Ferner müsste schon das Ei im Mutterleib erfahren und die junge Maurerbiene dann sich ihr ganzes Leben lang daran erinnert haben, dass, als ihre Mutter die Höhle für sie grub, diese den einen Teil des voll ihr befeuchteten und dann herausgegrabenen Sands in kleinen zusammenhängenden Massen um die Mündung der Höhle zu einem gegitterten freistehenden und gekrümmten Zylinder auf eine Weise aufbaute, welche nur dieser Art von 
Sandwespen eigen ist. Denn zur Zeit, als im darauffolgenden Jahre die junge Maurerwespe aus ihrer Höhle ging, war dieser vorläufig aufgebaute Eingangsturm längst nicht mehr da; die Mutter selbst hatte ihn wieder eingerissen, um mit den Materialien davon den übrigen Teil der Höhle, den die grünen Maden nicht einnahmen, wieder bis zum Eingang vollzustopfen; die junge Maurerwespe baut aber, wenn sie in der süssen Erinnerung an ihre Kindheit lebt, gerade einen gleichen kleinen hohlen Turm wie ihre Mutter, die sie nie sah noch sprach, einen vor ihrer Geburt gebaut und wieder zerstört hatte."

Beliebig vermehren liessen sich die Beispiele, aus denen die Unhaltbarkeit des Lamarckismus in jeder Form mit Sicherheit hervorgeht. Auch das Pflanzenreich bietet solche Beispiele in grosser Menge, so dass die Tatsache, dass in den letzten Jahren der Lamarckismus namentlich von botanischer Seite wieder hervorgeholt wird, geradezu als ein psychologisches Problem betrachtet werden muss.

Erinnert sei nur an all die mannigfachen Einrichtungen zur Verbreitung des Samens, welche in ihrer Gesamtheit Einrichtungen voraussetzen, die längst fertig sein müssen, wenn es zur Funktion kommt. Nur ein einziges Beispiel sei erwähnt. Der Samen der Balsamineen wird nach der Reife von den federnden Bestandteilen der Samenkapsel fortgeschleudert. Die Bestandteile der Samenkapsel müssen zu diesem Zwecke als gespannte Federn sich entwickeln. Die „Funktion“ d. h. das Losgehen dieses Apparates kann auf die Entstehung desselben keinen Einfluss ausüben. Und auch in den fortgeschleuderten Samenkörnern kann im Augenblick des Fortgeschleudertwerdens keine „Association“, keine „Erfahrung“, kein „Urteil“ geweckt werden über die Vorteile dieser Einrichtung, die sich erst später und nur dann geltend machen, wenn der Samen in einem geeigneten Boden aufgeht, der auf diese Weise gefunden wurde. Hier hört jede Möglichkeit auf, die Einrichtung in Lamarckistischem Sinne zu erklären, selbst wenn man dem Pauly'schen Prinzip dic weitgehendsten Zugeständnisse machen würde.

Schliesslich sei noch hingewiesen auf solche Fälle, in denen uns dasjenige unmittelbar entgegentritt, was von mir als „primäre Zweckmässigkeit" bezeichnet worden ist, ein Begriff, den ich am Beispiel der Linsenregeneration näher entwickelt habe. $\left.{ }^{1}\right)$

1) Vgl. meine Beiträge zur Kritik der Darwin'schen Lehre. p. 54 u. ff. 
Die Linse des Wirbeltierauges ist ja überhaupt ein Gebilde, welches allein genügen würde, die Ohnmacht des Lamarckistischen Prinzips nachzuweisen, sei es dass man dasselbe in Roux'schem Sinne, sei es in Pauly'schem Sinne modifiziert. Die Entstehung und Vervollkommnung ist nach Lamarckistischen Prinzipien nicht denkbar, weil wir uns nicht vorstellen können, dass die Linse um so besser ernährt wurde, je mehr Lichtstrahlen sie durchlassen und je mehr sie die Lichtstrahlen infolge ihres eigenartigen Baues sammeln konnte. Nicht die Linsenzellen empfinden das Licht, und am allerwenigsten erfahren sie etwas von der Wirkung eines etwa verbesserten Brechungsverhältnisses, denn nicht in der Linse, sondern erst auf der Retina kommt die das Bedürfnis befriedigende optische Wirkung der Linse zu stande. Es ist gar nicht abzusehen, wie die Linsenzellen erfahren sollen, ob sie das Licht richtig gebrochen haben und selbst wenn sie es erfahren würden, so könnten sie von dieser Erfahrung keinen Gebrauch machen, weil die Anordnung und Beschaffenheit der vorhandenen Linsenfasern nicht mehr verändert werden kann und weil es nicht vorstellbar ist, wie etwa das Linsenepitel durch Lieferung besserer Linsenfasern jene problematischen Erfahrungen sollte verwerten können.

Noch unverständlicher aber - wenn anders eine völlige Unverständlichkeit noch einer Steigerung fähig wäre - würde nach Lamarck'schen Prinzipien der Regenerationsvorgang sein, wie er sich bei Wassersalamandern nach Herausnahme der Linse vollzieht. Die schwarz pigmentierten Iriszellen wandeln sich in eine neue Linse um. Hier haben wir einen Funktionswechsel, dem der Lamarckismus in jeder Gestalt ebenso ratlos gegenübersteht wie der Darwinismus. Die Iriszellen müssen einen äusserst komplizierten Entwicklungsprozess durchmachen, bis die neue Funktion eintreten kann. Erst wenn alles fertig ist, kann die „Funktion“ zu stande kommen, die gar keine physiologische Funktion ist, und von deren Erfolg nicht einmal die Linse, sondern nur die Netzhaut etwas erfahren kann.

Mit gleicher Sicherheit zeigt sich die Unanwendbarkeit des Pauly'schen Prinzips gegenüber allen jenen Organen, in denen sich eine besondere Fähigkeit differenziert haben muss, der Einwirkung eines umgebenden Mediums sich speziell anzupassen, eines Mediums, das aber auf alle anderen Organe genau so gut 
einwirkt. Wie das Licht alle andern Augenteile nicht weniger trifft, als die Linse oder die Iris, so verhalten sich die Schallwellen den Knochen gegenüber, so dass in Lamarckistischem Sinne gar nicht zu verstehen ist, wie einzelne Knochen dazu kommen, sich zu Gehörknöchelchen umzubilden. Pauly sagt hierüber :

„Diese Knöchelchen, aus Teilen des Kiefergerüstes hervorgegangen, als, dessen Bestandteile sie ursprünglich analoge Funktionen und demnach auch Dimensionen besessen haben müssen, wie die heute noch unveränderten Kieferteile, haben dadurch, dass sie ihre ursprüngliche Funktion aufgaben und diejenige der Fortleitung von Schallwellen übernahmen, ganz neue zweckmässige Beiträge zu dieser Funktion zu leisten; während sie in ihrer ursprünglichen Tätigkeit bei Steigerung der Funktion sich zu vergrössern hatten, um die Funktion zu verstärken, hatten sie sich nun zu verkleinern, wemn sie die Schallwellen vollkommen fortpflanzen sollten. ${ }^{(1)}$

Aber die Schallwellen haben doch auf alle Knochen in gleicher Weise gewirkt, wie sollen daher einzelne sich besonders verkleinern durch diese „Funktion“, die gar keine Funktion ist, und die von allen anderen Knochen ebenso vollzogen wird? Wir können in solchen Fällen auch nicht der besonders gerichteten Aufmerksamkeit eine Rolle zuschreiben, etwa in der Weise, wie Pauly durch „die Verlegung der Aufmerksamkeit von einem Sinnesorgan auf das andere" ${ }^{\prime 2}$ ) die bessere Ausbildung des einen zu Gunsten des andern zu erklären sucht. Mag in der phylogenetischen Entwicklung des Gehörorgans die auf die Schallempfindungen gerichtete Aufmerksamkeit eine Rolle gespielt haben oder nicht - auf jeden Fall kann dies nur in der Weise geschehen sein, dass Schallempfindungen als solche mit immer grösserer Aufmerksamkeit zur Wahrnehmung kamen, nicht aber in der Weise, dass auf Tonempfindungen mehr oder weniger intensiv geachtet wurde, je nachdem die sie hervorrufenden Schallwellen mehr von diesem oder jenem Knochen geleitet worden waren, denn das Bewusstsein kann ja kein Datum darüber erhalten haben, durch welche Knochen diejenigen Schallwellen weitergeleitet worden sind, die eine Empfindung hervorgerufen haben. -

1) pag. 69 .

2) pag. 92,93 u. 212. 
Wir sehen also, dass der Lamarckismus vollständig versagt, welche Gestalt man ihm auch zu geben versucht. Gewiss ist Pauly völlig im Recht, wenn er für eine teleologische Auffassung des organischen Geschehens eintritt, aber durch 'die Lamarckistische Ausgestaltung der Teleologie erhält dieselbe eine Gestalt, die nachweislich falsch ist, denn es wird ihr dadurch eine Beschränktheit ihrer Wirkungsweise zuerteilt, die sie in Wirklichkeit nicht hat. So wenig wir auch im Einzelnen über die Wirkungsweise des teleologischen Prinzips wissen, dass dasselbe nicht in der von Pauly angenommenen Weise wirkt, ist vielleicht das Einzige, was wir mit Sicherheit von ihm sagen können. 
Von demselben Verfasser ist erschienen:

Beiträge zur Kritik der Darwin'schen Lehre. Georg Thieme, Leipzig. Mk. 2.-

Der gegenwärtige Stand des Darwinismus.

W. Engelmann, Leipzig. Mk. -.60.

Zur Psychologie des Erkennens.

W. Engelmannn, Leipzig. Mk. - .60

Mechanismus und Vitalismus,

Zweite vermehrte Auflage. Georg Thieme, Leipzig. Mk. 1.40

Ueber krankhafte Dissoziation der Vorstellungen. Joh. Ambr. Barth, Leipzig. Mk. 2.-

\section{Psychiatrie und Dichtkunst.}

J. F. Bergmann, Wiesbaden. Mk. 1.-

Klinische und kritische Beiträge zur Lehre von den

Sprachstörungen.

Veit \& Co., Leipzig. Mk. 2.40. 
Verlag von Ernst Reinhardt in München, Jägerstrasse 17.
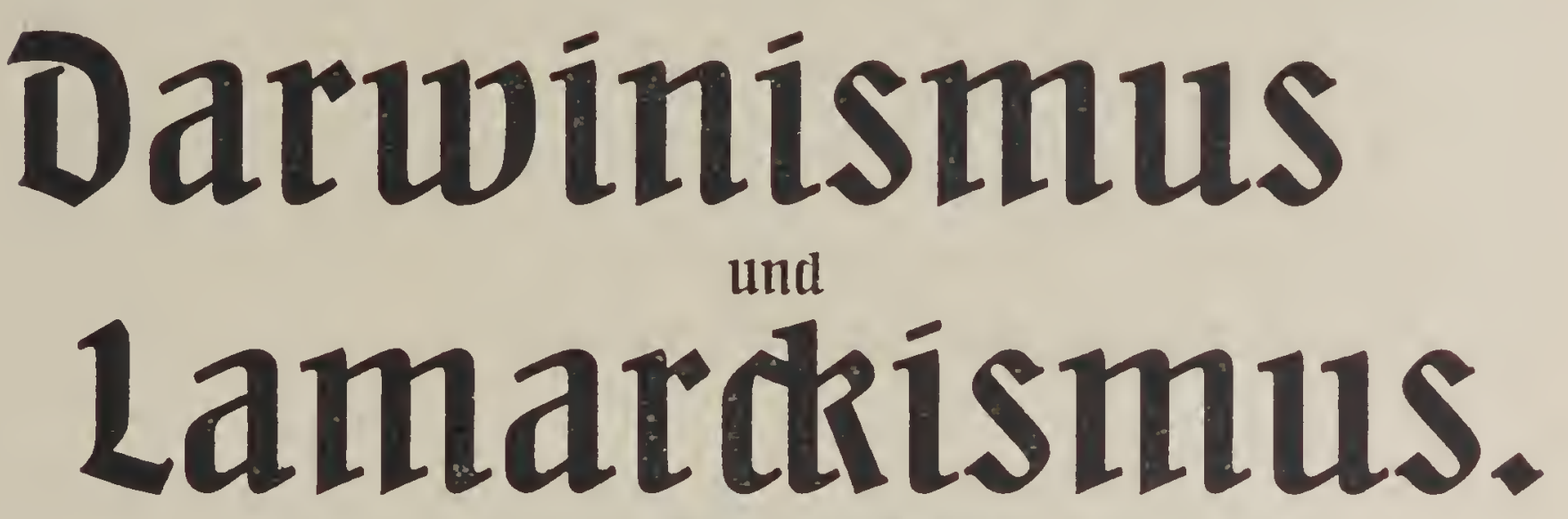

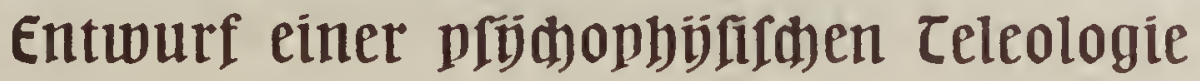

\section{Dr. Fuguft Pauly}

a o. Professor der angewandten Zoologie an der Universität München 352 Seiten. Mit 13 Illustrationen.

Preis brosch. Mk. 7.-, geb. Mk. 8.5̃0.

Der Streit in der Darwinschen Frage hat sich zuletzt immer mehr auf die Alternative zugespitzt "Darwinismus oder Lamarckismus", ohne dass es bis jetzt einer der beiden Parteien gelungen wäre, sie zu entscheiden. In dieser Alternative stehen sich zwei unvereinbare Prinzipien gegenüber: in der Zuchtwahllehre haben wir ein angeblich mechanistisches, nach dem berechtigten Vorw urf der Gegner ein mit dem Zufall arbeitendes Prinzip vor uns, im Lamarckismus dagegen die eigenen Kräfte des Organismus, also ein inneres Prinzip, eine aus dem Organismus wirkende Ursache.

In diesen Kampf bringt das vorliegende Buch die Entscheidung, indem es zwei Beweise zwingend durchführt. Der eine stellt mit unausweichbarer Logik das Zuchtwahlprinzip als wissenschaftlich unhaltbar hin, als Prinzip, welches, wenn man es vom Lamarckismus, mit dem es verbunden war, trennt, in seiner völligen Erklärungsunfähigkeit erscheint und sich als nicht mechanisch und ursachenlos herausstellt.

Der zweite Beweis richtet sich darauf, die in der Lamarckschen Theorie verborgene innere Ursache zu ermitteln. Dass diese im Organismus selbst liegende Ursache eine psychologische sein müsse, ist in der Polemik immer mehr hervorgetreten, ist schon von Lamarck erkannt worden und ebenso von den Neo. Lamarckianern. 
Diese Ursache verliert das den Naturforscher Befremdende dadurch, dass der Verfasser sie in seiner Analyse mit zwei ideellen Bestandteilen in Verbindung bringt, dem Kausalgesetz und dem Gesetz von der Erhaltung der Energie Hierdurch wird die organische Ursache zu einer psychophysischen, durch Experiment analysierbaren, also aus einer philosophischen zu einer naturwissenschaftlichen, welche alle Aussicht besitzt, eines Tages auch von der Analyse des Physikers ergriffen zu werden.

\section{Inbalts=ulibersicht.}

I. Kap. Allgemeines.

II. Kap. Psychologie des künstlichen Zweckmässigen.

III. Kap. Teleologie und Teleologien.

IV. Kap. Kritik des Darwinismus im engeren Sinne.

V. Kap. Dauerhaftigkeit der Zuchtwahllehre. Kritik ihrer Wirkung mit Bemerkungen ïber Philosophie. Ueberleitung zu Lamarck. Anzeichen für den Untergang des Falschen und für das Aufblühen des Wahren.

VI. Kap. Jean-Baptiste-Pierre-Antoine de Monet, Chevalier de Lamarck. VII. Kap. Wilhelm Roux.

VIII. Kap. Anhang: Ueber die rudimentären Organe als Beweismittel des Lamarckismus.

IX Kap. Der Begriff des Mittels.

X. Kap. Mechanismus und Vitalismus.

XI. Kap. Pflanzenpsychologie.

XII. Kap. Körper und Nervenpsychologie der Tiere und des Menschen. XIII. Kap. Ueber die teleologische Reaktionsfähigkeit der Vogelfeder.

XIV. Kap. Die Annäherung der Fachpsychologie an unser Problem. Unabhängikeit der aufsteigenden organischen Entwicklung von einer vorausbestimmenden Ursache. Unabhängigkeit de organischen Fortschrittes von objektiven koinzidentellen Bedingungen. Freiheit der Kausalität aller Wesen. Weltzweck im Ueberblick des Ganzen. Unvermeidlichkeit geistiger Potenzierung. Technische Logik in den Reaktionen. Kosmische Gültigkeit logischer Gesetze.

Nachwort. 
Verlag von Ernst Reinhardt, München; Jägerstrasse 17.

\section{Vom Nebelfleck zum Menschen.}

Eine gemeinverständliche Entwicklungsgeschichte des Naturganzen nach den neuesten Forschungsergebnissen

von

Dr. Ludwig Reinbardt.

\section{Die Geschidte der Erde.}

Mit gegen 200 Abbildungen im Text, 17 Volltafeln und 3 geolo. gischen Profiltafeln nebst farbigem Titelbild von A. Mardks.

6r. $8^{0}$ in elegantem Leinenband Preis mk. 8.50.

\section{Inhalt:}

1. Wie das Weltbild entstand. II. Die Sternenwelt. III. Unser Sonnensystem. IV. Die Erde und der Mond. V. Die Kometen und Meteore. VI. Die Erstarrungsgesteine der Erde. VII. Der Vulkanismus. VIII. Die Schichtgesteine. IX. Die Gebirgsbildung. $X$. Wasser und Land. XI. Der Kreislauf des Wassers. XII. Die Verwitterung der Erdoberfläche. XIII. Die Abtragung des Festlandes.

Es ist schon oft von Fachleuten bedauert worden, dass es im Deutschen keine gute populäre Geologie gibt, die auf den neuesten wissenschaftlichen Forschungen fusst und das ungeheure Material, das in wissenschaftlichen Zeitschriften und Monographien vergraben ist, in einer für den gebildeten Leser genussvollen Darstellung wiedergibt, ohne in literarische Phantastereien zu verfallen. Diese schwierige Aufgabe zu lösen war wohl niemand besser berufen als der Verfasser, dessen im vorigen Tahre erschienenes Buch "Der Mensch zur Eiszeit in Europa" bei Laien und Fachgelehrten eine geradezu begeisterte Aufnahme gefunden hat Was man an diesem Werke lobt: eine gründliche Quel!enkenntnis, wissenschaftliche Behandlung des Stoffes und eine klare, stilistisch vollendete Darstellung, das zeichnet atch dieses neueste Werk aus. 


\section{Der Mensch zur Eiszeit in Europa}

und seine Kulturentwicklung bis zum Ende der Steinzeit Dr. Ludwig Reinhardt.

Mit 186 Abbildungen und farbigem Umschlag nach Aquarell von A. Thomann. VIII und 504 Seiten gr. 8".

Preis brosch. Mk. 7.—, elegant geb. Mk. 8.50.

\section{Inhaltsverzeichnis:}

I. Der Mensch zur Tertiärzeit. II. Die Eiszeit und ihre geologischen Wirkungen. III. Der Mensch während den ersten Zwischeneiszeiten. IV. Der Mensch der letzten Zwischeneiszeit. V. Der Mensch der frühen Nacheiszeit. VI. Die Uebergangsperiode von der älteren zur jüngeren Steinzeit. VII. Die jüngere Steinzeit und ihre materiellen Kulturerwerbungen. VIII. Die Germanen als Träger der megalithischen Kultur. IX. Die Entwicklung der geistigen Kultur am Ende der Steinzeit. X. Steinzeitmenschen der Gegenwart. XI. Niederschläge aus alter Zeit in Sitten und Anschauungen der geschichtlichen Europäer.

\section{Urteile der Presse:}

"Geologisches Zentralblatt66 vom 1. Aug. 1906. Das Buch ist unstreitig das beste was über diesen Gegenstand vorhanden ist.

"Prometheus", Nr. 853: Das vorliegende Buch bietet, trotzdem es einen Stoff behandelt, der seinem wesentlichen Umfange nach schon wiederholt in allgemein verständlicher Weise erörtert worden ist, eine grosse Reihe neuer Gesichtspunkte. Es ist so fesselnd und anschaulich geschrieben, dass es lebhaft allen denjenigen empfohlen werden kann, welche über die Vergangenheit und die Geschichte des Menschengeschlechts eine eingehende und doch in keiner Beziehung phantastische Schilderung im Zusammenhang lesen wollen. Die umfassenden Quellenstudien des Verfassers ermöglichen es ihm, den reichen Stoff, ohne mit Einzelheiten zu ermüden, so fesselnd und dabei einfach darzustellen, dass die Lektuire jedem denkenden Menschen einen grossen Genuss bereiten muss.

„Petermanns Mitteilungen“", 1906, Heft 8: ... Es ist zweifellos, dass er ein Buch geschaffen hat, das dieser Aufgabe vollkommen gerecht wird. Mit grossem Fleisse ist die Literatur, sind die Funde bis in die neueste Zeit hinein zum Unterbau der Darstellung verwendet, die selbst durch zahlreiche Illustrationen unterstützt, ein lebendiges und - für popularisierende Arbeiten das schwerste - auch ein wissenschaftliches Bild von der Menschwerdung in ihren ältesten tertiären Spuren bis zum Ende der reinen Steinzeit gibt, das nie den realen Boden der Forschung verlässt. 

